\title{
TIME-DOMAIN, SHALLOW-WATER HYDROELASTIC ANALYSIS OF VLFS ELASTICALLY CONNECTED TO THE SEABED
}

\author{
A. E. Karperaki ${ }^{1}$, K. A. Belibassakis ${ }^{1}$, T. K. Papathanasiou ${ }^{2}$ \\ ${ }^{1}$ School of Naval Architecture and Marine Engineering \\ National Technical University of Athens \\ Iroon Polytechniou St. 9, Zografou Campus 15773 Athens, Greece \\ e-mail: karperaki.ang@gmail.com, kbel@fluid.mech.ntua.gr \\ ${ }^{2}$ Department of Mechanics, Sch. of Applied Mathematical and Physical Science, \\ National Technical University of Athens, Iroon Polytechniou St. 9, \\ Zografou Campus 15773 Athens, Greece, e-mail: papathth@gmail.com \\ now at: DICAM, University of Trento, via Mesiano 77, 38123 Trento, Italy
}

\begin{abstract}
In order to ensure the safe operation of a VLFS, a combination of mooring, breakwater and other motion reducing systems is employed. In the present work, the transient hydroelastic response of a floating, thin elastic plate, elastically connected to the seabed, is examined. The plate is modelled as an Euler-Bernoulli strip, while the linearized shallow water equations are used for the hydrodynamic modelling. Elastic connectors are approximated by a series of simple spring-dashpot systems positioned along the strip. A higher order finite element scheme is employed for the calculation of the hydroelastic response of the strip-connector configuration, over the shallow bathymetry. After the definition of the initial-boundary value problem, its variational form is derived and discussed. Next, on the basis of the aforementioned formulation, an energy balance expression is obtained. The effect of variable bathymetry on the response of a two connector-strip system, is examined by means of three seabed profiles, featuring a flat bottom, an upslope and a downslope environment. For the flat bottom case, the strip response mitigating effect exerted by the employment of two and three elastic connectors is considered. Finally, by means of the derived energy balance equation, the energy exchange is monitored, providing a valuable insight into the transient phenomena that take place in the studied configurations.
\end{abstract}

Keywords: VLFS, transient hydroelastic analysis, variable bathymetry, shallow water, FEM 


\section{Introduction}

In the past decades, due to the advances in marine technology, the hydroelastic response of Very Large Floating Structures (VLFSs) has received great scientific attention. Population densification in coastal areas, along with the increasing work load in major ports, has led to costly land reclamation solutions in order to accommodate the need for commercial space, necessary for industrial growth [1]. Compared to expanding industrial zones inland or resulting to environmentally hostile and costly land reclamation solutions, the employment of VLFS as operational docks constitutes an attractive alternative. Pontoon type VLFSs are essentially floating plates of large horizontal dimensions resting on the water surface [2]. With horizontal dimensions stretching from tens to hundreds of meters, VLFSs provide extended floor span, highly desirable for various applications ranging from storage, docking and military operation platforms to recreational facilities and floating airport and helicopter bases $[1,2]$. Moreover, the ability to moor the structures at safe distances from the shore makes them suitable for the accommodation of socially sensitive facilities, such as power and sewage treatment plants [1-5].

The large length to thickness ratio of VLFSs makes elastic deformation dominant under ocean wave action. Hence, the extensive study and comprehension of hydroelastic effects is essential in the development of robust VLFSs' design codes. Due to their small rigidity, pontoon type VLFSs are most commonly modelled as thin, elastic, floating plates of either negligible or non-negligible draft. Commonly the classical Kirchhoff plate theory is used for the approximation of the strip deflection [6-7], while some works consider higher -order [8] and non-linear strip [9] theories.

Most tools developed for the study of hydroelastic effects employ either frequency domain or time-domain techniques. Frequency domain tools employ Galerkin schemes [7], Green function methods [10] and eigenfunction expansion approaches [11]. However, the treatment and analysis of transient phenomena, characterised by steep wave fronts, and strong nonlinearity effects, requires time domain methods. Analysis tools in the time domain include direct integration schemes [12-13] and Fourier transform techniques [14-15]. Considering long wave excitation, Sturova [16] developed an eigenfunction expansion technique for the calculation of the hydroelastic response of thin heterogeneous plates. In the same line of work, Papathanasiou et al [17] proposed a higher order finite element scheme for the 
solution of the initial-boundary value hydroelastic problem of a thin plate floating over mildly sloped bathymetry in shallow water conditions.

Pontoon type VLFSs are suitable for calm waters and are usually moored nearshore. The proximity to coastal areas and the large horizontal dimensions make variable bathymetry effects important. In [18] the effects of a sloping sea bed are considered, while a fast-multipole method is developed in [19] to account for variable bathymetry. Belibassakis \& Athanassoulis [20] have developed a coupled mode method for the calculation of the hydroelastic response of a floating, thin plate over general bathymetry.

In order to avoid drift off and reduce vibration effects of a VLFS, a combination of mooring, breakwater and other motion reducing systems is employed [1-3]. The choice of the response mitigating system is dictated by the allowable displacement values for the given configuration. Negata [21] and Seto \& Ochi [22] showed that the motion of a floating plate surrounded by bottom-founded breakwaters is considerably reduced in the case of incoming long waves. Numerical studies have confirmed that the gravity type breakwater system is highly effective in reducing both drift forces on the floating structure and its hydroelastic response [23-24].

On the other hand, bottom-founded breakwaters have a profound environmental impact, as they disrupt ocean currents, and costly construction. Alternative breakwater systems, like the box-like floating breakwater [3], have been proposed as eco-friendly alternatives. The need to mitigate the hydroelastic response of floating bodies has also led to the development of auxiliary structural elements acting as motion reducing mechanisms. Such devices, attached at the free edges of the floating structure, are able to dissipate the incoming wave energy and achieve the necessary hydroelastic response mitigation. The devices range from submerged vertical or horizontal plates [25-26], acting as reflectors, to air cushions [27]. In order to derive the optimal configuration for a given structure and environmental conditions, computationally intensive, parametric studies must be carried out. Khabakhpasheva \& Korobkin [28] underline the need for a simple model able to capture the effect of the motion reducing device on the dynamic response of the structure. In the same work, the response mitigating effect of an elastic spring, connecting one of the free edges of the floating strip to the seabed, is studied among other systems. Finally, Karmakar \& Guedes Soares [29] study the scattering of gravity waves by a moored elastic strip, floating over shallow bathymetry, in the frequency domain. In [29] a thorough 
analysis of the vertical strip deflection, bending moment, strain shear force and spatial distribution for moored configurations under harmonic excitation is presented.

In the present work, the time-domain hydroelastic response of a thin, elastic, floating plate, elastically connected to the sea bed, is examined. The plate is modelled as an Euler-Bernoulli strip, while the linearized shallow water equations are used for the hydrodynamic modelling. The main novelty of the present contribution, compared to the previous work carried out by the authors [17], lays on the inclusion of multiple elastic connectors in the developed shallow-water, time-domain model. The elastic connectors are represented by simple spring-dashpot systems distributed along the structure. The present study also considers the effect of the number, arrangement, stiffness and damping coefficients of the connectors on the resulting transient hydroelastic response of the strip-connector configuration, floating over shallow waters. The investigation finds important applications in a number of fields, such as the design of mooring systems [29], the vibration reduction of a floating structure [30] and wave energy harvesting [31]. The numerical solution is calculated by means of a higher order finite element scheme.

In Sect. 2, the initial-boundary value problem is formulated. Next, in Sect. 3 the variational form of the above problem is given. Subsequently, the energy balance expression is derived from the variational form, while the employed finite element scheme is briefly introduced. Finally, in Sect. 4 a series of numerical results is presented. In order to explore the effects of bathymetry, three seabed profiles are defined. Namely, a flat bottom, an upslope and a downslope environment were considered. For the flat bottom case, the response reducing effects of elastic strip configurations employing two and three connectors, are studied and compared against the freely floating case. Strip deflection and bending moment distributions in given time instances are plotted for various elastic connector parameters. Finally, the energy exchange within the system is monitored by means of the energy balance expression, providing a valuable insight into the physical phenomenon and the effectiveness of the studied configurations.

\section{Governing Equations}

In this section, the hydroelastic problem of a thin, floating, strip that is elastically connected to the seabed, is presented. Shallow-water conditions are assumed in the following analysis. The general formulation of the above problem, for a freely- 


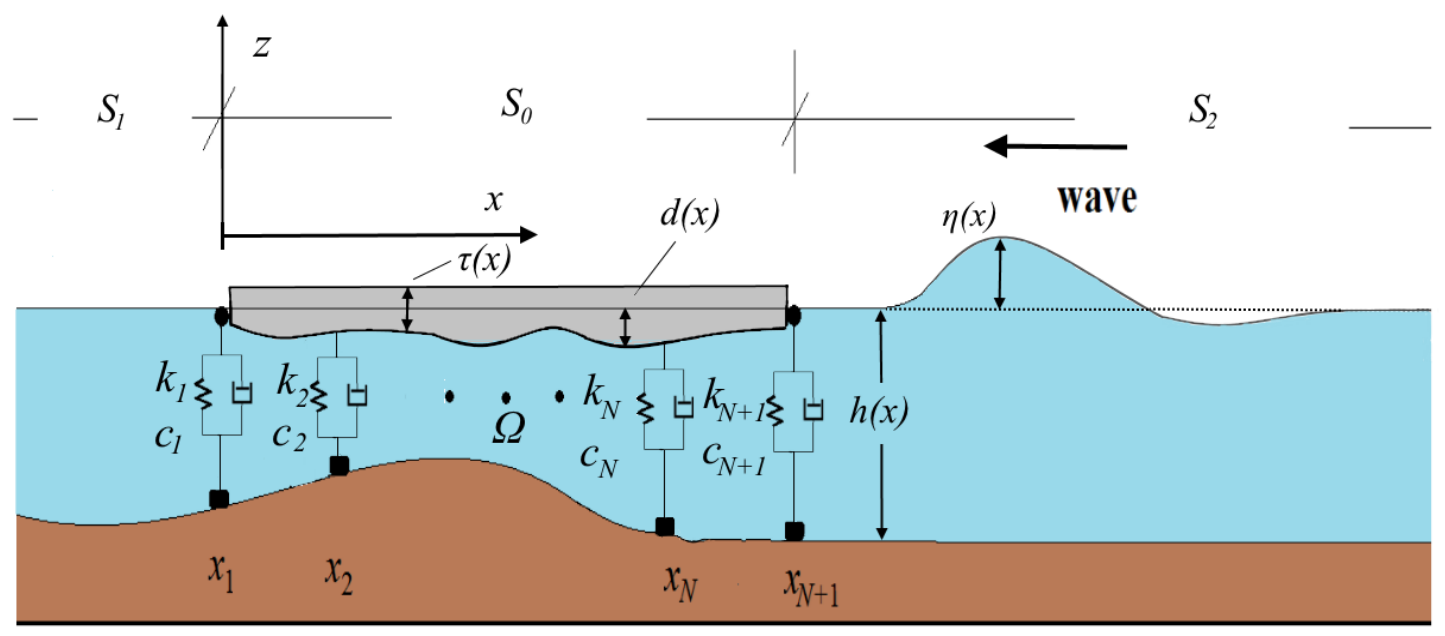

Figure 1 A floating elastic strip with multiple elastic connectors along its length.

floating elastic strip, has already been presented in Sturova [16] and Papathanasiou et al [17]. In the present contribution, the strip is assumed to be elastically connected to the bottom boundary, at both edges, while additional $N-1$ elastic connectors are distributed along the strip length (see Fig. 1). A Cartesian coordinate system is introduced. The horizontal axis $x$ coincides with the mean water level, while the vertical axis $z$ is pointed upwards. The plate extends infinitely in the direction vertical to the page, hence allowing the treatment of the floating body configuration in the $x z$ plane.

The upper surface elevation is denoted by $\eta(x, t)$. The thin, elastic strip of length $L$, thickness $\tau(x)$ and density $\rho_{p}$ is resting over a layer of water with density $\rho_{w}$. The fluid layer is contained in the domain $\Omega:(-\infty<x<\infty,-b<z<\eta)$ where the depth function is given by $b(x)=h(x)-d(x)$, with $h(x)$ being the depth measured up to the mean water level and $d(x)=\tau(x) \rho_{p} / \rho_{w}$ the plate draft. The horizontal extent of the domain is decomposed into subregion $S_{0}: 0<x<L$, where the hydroelastic coupling takes place, and the free fluid surface subregions, $S_{1}:(-\infty, 0]$ and $S_{2}:[L,-\infty)$. In the middle region $S_{0}$, the plate deflection and the free surface elevation coincide. The velocity potential functions, defined in each sub domain, are denoted as $\varphi_{i}, i=0,1,2$ respectively. Assuming a thin body, the Euler-Bernoulli beam theory can be employed for the approximation of the floating strip hydroelastic response. The resulting system of equations, valid in $\Omega$, becomes 


$$
\begin{aligned}
& m(x) \partial_{t t} \eta+\partial_{x x}\left[D(x) \partial_{x x} \eta\right]+\rho_{w} g \eta+\rho_{w} \partial_{t} \varphi_{0}=\sum_{n=2}^{N} \delta\left(x-x_{n}\right)\left(k_{n} \eta+c_{n} \partial_{t} \eta\right), x \in S_{0}, \\
& \partial_{t} \eta+\partial_{x}\left[b(x) \partial_{x} \varphi_{0}\right]=0, \quad x \in S_{0}, \\
& \partial_{t t} \varphi_{1}-g \partial_{x}\left[b(x) \partial_{x} \varphi_{1}\right]=0, \quad x \in S_{1}, \\
& \partial_{t t} \varphi_{2}-g \partial_{x}\left(b(x) \partial_{x} \varphi_{2}\right)=0, x \in S_{2},
\end{aligned}
$$

where $g$ is the acceleration of gravity and $m(x)=\rho_{p} \tau(x)$ is the plate mass per unit length. The Dirac function is denoted by $\delta$. The flexural rigidity of the plate is $D(x)=E \tau(x)^{3} / 12\left(1-v^{2}\right)$, with $E$ being the Young's modulus, $v$ the Poisson's ratio of the plate material. Furthermore, it is assumed that $t / L \ll 1$ in order to comply with the Kirchhoff thin plate theory. Finally, the strip is connected with the seabed, at $x_{n}$ horizontal locations, by elastic connectors with stiffness $k_{n}$, and damping coefficients $c_{n}$ for $n=1, \ldots, N+1$, represented by simple spring-dashpot systems. Equation (1) accounts for the deflection of the elastic strip, according to the Kirchhoff plate theory assumptions, resting on a fluid layer described by the linearized shallow water equations. The present model incorporates inertial and flexural effects by means of the terms $m(x) \partial_{t t} \eta$ and $\partial_{x x}\left[D(x) \partial_{x x} \eta\right]$, respectively. The classical thin plate model is augmented by the hydroelastic coupling terms $\rho_{w} g \eta$ and $\rho_{w} \partial_{t} \varphi_{0}$, rising from the linearized dynamic condition at the upper surface boundary of the middle region $S_{0}$.

The forcing term in the right hand side of Eq. (1) accounts for the collective restoring effect of the elastic connectors distributed along the strip length $(n=2, \ldots, N)$, excluding edge connectors. Notably, the restoring effect of the connectors positioned at the free edges of the strip is accounted by the imposed non-zero shear force boundary conditions at the strip edges and is thus not included in the aforementioned forcing term. Moreover, Eq. (2) expresses mass conservation in the water region, under the plate, while Eqs. (3) and (4) are derived through a simple algebraic manipulation of the linearized shallow water equations, modelling long wave propagation in the free water surface subregions $S_{i}, i=1,2$. For the given subregions, it equivalently holds that $\eta_{i}=-g^{-1} \partial_{t} \varphi_{i}, i=1,2$, from the integration of the equation of motion in the respective regions. Hence, the upper surface elevation in the halfstrips is directly derived from the corresponding velocity potential functions. 
The system of Eqs. (1)-(4) is supplemented by the following conditions at infinity,

$$
\partial_{x} \varphi_{1}(x \rightarrow-\infty, t)=0 \text { and } \partial_{x} \varphi_{2}(x \rightarrow \infty, t)=0
$$

implying quiescence in the far field. At the interfaces between subregions mass and energy conservation is assumed, yielding the following matching conditions, $b\left(0^{-}\right) \partial_{x} \varphi_{1}\left(0^{-}, t\right)=b\left(0^{+}\right) \partial_{x} \varphi_{0}\left(0^{+}, t\right)$ and $b\left(L^{+}\right) \partial_{x} \varphi_{2}\left(L^{+}, t\right)=b\left(L^{-}\right) \partial_{x} \varphi_{0}\left(L^{-}, t\right)$ $\partial_{x} \varphi_{1}\left(0^{-}, t\right)=\partial_{x} \varphi_{0}\left(0^{+}, t\right)$ and $\partial_{x} \varphi_{0}\left(L^{-}, t\right)=\partial_{x} \varphi_{2}\left(L^{+}, t\right)$

At the free strip edges, located at $x=0$ and $x=L$, zero-moment and non-zero shear force conditions are imposed,

$D(0) \partial_{x x} \eta=0$ and $D(0) \partial_{x x} \eta=-k_{1} \eta-c_{1} \partial_{t} \eta$ at $x=0$, $D(L) \partial_{x x} \eta=0$ and $D(L) \partial_{x x x} \eta=k_{N+1} \eta+c_{N+1} \partial_{t} \eta$ at $x=L$.

Initially at $t=0$ the plate is at rest, while a free water surface disturbance, denoted by $S(x)$, begins to propagate in subregion $S_{2}$. Thus, the conditions that complete the initial-boundary value problem are given as $\eta(x, 0)=\partial_{t} \eta(x, 0)=\partial_{x} \varphi_{0}=0, x \in S_{0}$ and $\varphi_{1}=\partial_{t} \varphi_{1}=0, x \in S_{1} \varphi_{2}=0, \partial_{t} \varphi_{2}=-S(x), x \in S_{2}$.

Using the following non-dimensional variables $\tilde{x}=x L^{-1}, \tilde{\eta}=\eta L^{-1}, \quad \tilde{t}=g^{1 / 2} L^{-1 / 2} t$, $\tilde{\varphi}_{i}=g^{-1 / 2} L^{-3 / 2} \varphi_{i}$, for $i=0,1,2$, the initial-boundary value problem under consideration is rewritten (after dropping tildes)

$$
\begin{aligned}
& M(x) \partial_{t t} \eta+\partial_{x x}\left[K(x) \partial_{x x} \eta\right]+\eta+\partial_{t} \dot{\varphi}_{0}=\sum_{n=2}^{N} \delta\left(x-x_{n}\right)\left(\widehat{k}_{n} \eta+\widehat{c}_{n} \partial_{t} \dot{\eta}\right), x \in S_{0}, \\
& \partial_{t} \eta+\partial_{x}\left[B(x) \partial_{x} \varphi_{0}\right]=0, \quad x \in S_{0}, \\
& \partial_{t t} \varphi_{1}-\partial_{x}\left[B(x) \partial_{x} \varphi_{1}\right]=0, \quad x \in S_{1}, \\
& \partial_{t t} \varphi_{2}-\partial_{x}\left[B(x) \partial_{x} \varphi_{2}\right]=0, \quad x \in S_{2},
\end{aligned}
$$

where the following non-dimensional quantities are involved,

$$
M(x)=m(x) \rho_{w}^{-1} L^{-1}, K(x)=D(x) \rho_{w}^{-1} g^{-1} L^{-4} \text { and } B(x)=b(x) L^{-1} .
$$


The corresponding interface conditions become

$$
\begin{aligned}
& B\left(0^{-}\right) \partial_{x} \varphi_{1}\left(0^{-}, t\right)=B\left(0^{+}\right) \partial_{x} \varphi_{0}\left(0^{+}, t\right), B\left(1^{-}\right) \partial_{x} \varphi_{0}\left(1^{-}, t\right)=B\left(1^{+}\right) \partial_{x} \varphi_{2}\left(1^{+}, t\right), \text { and } \\
& \dot{\varphi}_{1}\left(1^{-}, t\right)=\dot{\varphi}_{0}\left(1^{+}, t\right), \partial_{t} \varphi_{0}\left(1^{-}, t\right)=\partial_{t} \varphi_{2}\left(1^{+}, t\right)
\end{aligned}
$$

while the non-dimensional boundary conditions read as follows

$$
K(0) \partial_{x x} \eta=0 \text { and } K(1) \partial_{x x} \eta=0 \text { at } x=0 \text { and }
$$

$K(0) \partial_{x x x} \eta=-\widehat{k}_{1} \eta(0, t)-\widehat{c}_{1} \partial_{t} \eta(0, t)$ and

$$
K(1) \partial_{x x x} \eta=\widehat{k}_{N+1} \eta(1, t)+\widehat{c}_{N+1} \partial_{t} \eta(1, t) \text { at } x=1 \text {. }
$$

In the above equations $\hat{k}_{n}=k_{n}\left(\rho_{w} g\right)^{-1}$ and $\hat{c}_{n}=c_{n} g^{1 / 2} L^{-1 / 2}\left(\rho_{w} g\right)^{-1}$, for $n=1,2, \ldots, N+1$, are the non-dimensional connector stiffness and damping coefficients. For simplicity in presentation, the hat notation is omitted in the following analysis.

\section{Variational Formulation}

The variational form of the previously defined transient hydroelastic problem is derived and discussed in the present section. For the derivation of the variational formulation of the problem the same standard process is followed as in Papathanasiou et al [17]. The reader is directed to the given work for a more detailed account. Concisely, it is mentioned that Eqs. (6)-(9) are multiplied by the weight functions $v \in H^{2}\left(S_{0}\right),-w_{0} \in H^{1}\left(S_{0}\right), w_{1} \in H^{1}\left(S_{1}\right)$, and $w_{2} \in H^{1}\left(S_{2}\right)$, respectively (where $H$

denotes the Sobolev spaces in the corresponding intervals). After performing integration by parts and adding the resulting equations, the following variational problem is defined,

Find $\eta(x, t), \varphi_{0}(x, t), \varphi_{1}(x, t)$ and $\varphi_{2}(x, t)$ such that for every $v \in H^{2}\left(S_{0}\right)$, $-w_{0} \in H^{1}\left(S_{0}\right), w_{1} \in H^{1}\left(S_{1}\right)$ and $w_{2} \in H^{1}\left(S_{2}\right)$ it holds that

$$
\begin{aligned}
\int_{0}^{1} M v \partial_{t t} \eta d x+\int_{0}^{1} v \partial_{t} \varphi_{0} d x-\int_{0}^{1} w_{0} \partial_{t} \eta d x+\int_{-\infty}^{0} w_{1} \partial_{t t} \varphi_{1} d x+\int_{1}^{\infty} w_{2} \partial_{t t} \varphi_{2} d x+ \\
+a(\eta, v)+b_{0}\left(\varphi_{0}, w_{0}\right)+b_{1}\left(\varphi_{1}, w_{1}\right)+b_{2}\left(\varphi_{2}, w_{2}\right)+q(\eta, v)+c\left(\partial_{t} \eta, v\right)=0
\end{aligned}
$$


where the bilinear functionals are given by

$$
\begin{aligned}
& q(\eta, v)=\sum_{n=1}^{N+1} v\left(x_{n}\right) k_{n} \eta\left(x_{n}, t\right) \text { and } \\
& c(\dot{\eta}, v)=\sum_{n=1}^{N+1} v\left(x_{n}\right) c_{n} \partial_{t} \eta\left(x_{n}, t\right),
\end{aligned}
$$

and as defined in [17],

$a(\eta, v)=\int_{0}^{1}\left(K \partial_{x x} v \partial_{x x} \eta+v \eta\right) d x$

$b_{0}\left(\varphi_{0}, w_{0}\right)=\int_{0}^{1} \partial_{x} w_{0} B \partial_{x} \varphi_{0} d x$

$b_{1}\left(\varphi_{1}, w_{1}\right)=\int_{-\infty}^{0} \partial_{x} w_{1} B \partial_{x} \varphi_{1} d x$

$b_{2}\left(\varphi_{2}, w_{2}\right)=\int_{1}^{\infty} \partial_{x} w_{2 x} B \partial_{x} \varphi_{2} d x$

\subsection{Energy balance considerations}

Following Ref. [17] an energy balance equation is derived from the variational formulation Eq. (15). The above result is subsequently used in order to study the energy exchange between the defined subregions in the presence of non-conservative restoring forces.

In order to derive the energy conservation principle, we set $v=\partial_{t} \eta, w_{0}=\partial_{t} \varphi_{0}$, $w_{1}=\partial_{t} \varphi_{1}$ and $w_{2}=\partial_{t} \varphi_{2}$ in Eq. (15). The substitution is valid under sufficient regularity assumptions for the weak solution and the definition of the weight functions given above. Hence, Eq. (15) is transformed into the following

$$
\begin{array}{r}
\frac{1}{2} \frac{d}{d t}\left[\int_{0}^{1} M\left(\partial_{t} \eta\right)^{2} d x+\int_{-\infty}^{0}\left(\partial_{t} \varphi_{1}\right)^{2} d x+\int_{1}^{\infty}\left(\partial_{t} \varphi_{2}\right)^{2} d x+a(\eta, \eta)+b_{0}\left(\varphi_{0}, \varphi_{0}\right)+\right. \\
\left.+b_{1}\left(\varphi_{1}, \varphi_{1}\right)+b_{2}\left(\varphi_{2}, \varphi_{2}\right)+q(\eta, \eta)+2 \int_{0}^{t} c\left(\partial_{s} \eta, \partial_{s} \eta\right) d t\right]=0,
\end{array}
$$

where, Eqs. (16a, b) take the form

$$
q\left(\eta, \partial_{t} \eta\right)=\frac{1}{2} \frac{d}{d t} \sum_{n=1}^{N+1} k_{n} \eta^{2}\left(x_{n}, t\right)=\frac{1}{2} \frac{d}{d t} q(\eta, \eta) \quad \text { and }
$$


$c\left(\partial_{t} \eta, \partial_{t} \eta\right)=\sum_{n=1}^{N+1} c_{n}\left[\partial_{t} \eta\left(x_{n}, t\right)\right]^{2}$

while, after substitution, the functionals of Eqs. (16c-f) are rewritten as in [17].

In Eqs. (17) and (18b) $s$ denotes a dummy variable. Equation (17) expresses the energy conservation principle for the studied system. The quantity $\mathcal{E}(t)$

$$
\begin{gathered}
\mathcal{E}(t)=\int_{0}^{1} M\left(\partial_{t} \eta\right)^{2} d x+\int_{-\infty}^{0}\left(\partial_{t} \varphi_{1}\right)^{2} d x+\int_{1}^{\infty}\left(\partial_{t} \varphi_{2}\right)^{2} d x+a(\eta, \eta)+b_{0}\left(\varphi_{0}, \varphi_{0}\right)+ \\
+b_{1}\left(\varphi_{1}, \varphi_{1}\right)+b_{2}\left(\varphi_{2}, \varphi_{2}\right)+q(\eta, \eta)+2 \int_{0}^{t} c\left(\partial_{s} \eta, \partial_{s} \eta\right) d t
\end{gathered}
$$

i.e. the quantity in the brackets in the left-hand side of Eq. (17) should remain constant in time, and equal the energy provided by the initial free surface disturbance, $\mathcal{E}(t)=\mathcal{E}(0)$ for every $0 \leq t \leq T$. The above energy balance equation provides a valuable tool in the study of the hydroelastic wave propagation in the defined stripconnector system. When the excitation reaches the strip, the strain and kinetic energy of the plate will increase and eventually vanish as the wave exits the structure and a state of rest is reached. The study of the initial excitation energy $(\mathcal{E}(0))$ conversion, as the pulse propagates in $S_{0}$, in correlation with the configuration material and geometry parameters, is indicative of the elastic connector effects on the strip response. Following that line of thought, it is interesting to examine the quantities appearing in the energy balance equation (19). In the free water surface subregions $S_{i}, i=1,2$ the total energy is defined as the sum of the kinetic and potential energy of the water column given respectively as,

$$
\begin{aligned}
& E_{K 2}(t)=\frac{1}{2} \int_{1}^{\infty}\left(\partial_{t} \varphi_{2}\right)^{2} d x \text { and } E_{P 2}(t)=\frac{1}{2} \int_{1}^{\infty} B\left(\partial_{x} \varphi_{2}\right)^{2} d x, \text { for } S_{2}, \\
& E_{K 1}(t)=\frac{1}{2} \int_{-\infty}^{0}\left(\partial_{t} \varphi_{1}\right)^{2} d x \text { and } E_{P 1}(t)=\frac{1}{2} \int_{-\infty}^{0} B\left(\partial_{x} \varphi_{1}\right)^{2} d x, \text { for } S_{1} .
\end{aligned}
$$

Additionally, the kinetic and strain energy of the strip are given by the following terms,

$$
E_{K}(t)=\frac{1}{2} \int_{0}^{1} M \partial_{t} \eta^{2} d x
$$


and $E_{S}(t)=\frac{1}{2} \int_{0}^{1} K\left(\partial_{x x} \eta\right)^{2} d x$

The total fluid energy in the subregion $S_{0}$ is given as follows,

$$
E_{P}(t)=\frac{1}{2} \int_{0}^{1}\left[B\left(\partial_{x} \varphi_{0}\right)^{2}+\eta^{2}\right] d x
$$

The quantity of Eq. (23) consists of the kinetic fluid energy in the middle subregion and the potential energy due to elastic strip deflection. Furthermore, the elastic potential energy of the employed $N+1$ connectors is given by

$$
W(t)=\frac{1}{2} \sum_{n=1}^{N+1} k_{n} \eta^{2}\left(x_{n}, t\right),
$$

while the energy dissipation due to connector damping is expressed as

$$
C(t)=\sum_{n=1}^{N+1} \int_{0}^{t} c_{n}\left[\partial_{s} \eta\left(x_{n}, s\right)\right]^{2} d s
$$

Integrating Eq. (19) with respect to time from $t=0$ to $t=T$, and using the fact that $\mathcal{E}(t)=\mathcal{F}_{0}$, the following holds

$$
\int_{0}^{T} \mathcal{E}(t) d t=\mathcal{E}_{0} T
$$

where $\mathcal{F}_{0}$ is the initial excitation energy, expressed as the sum of potential and kinetic energy of the water column, provided by the imposed free surface disturbance $S(x)$ in the right halfstrip $S_{2}$. Equation (26) is written in a more convenient form as,

$$
\sum_{l=1}^{2}\left(\bar{E}_{K_{l}}+\bar{E}_{P_{l}}\right)+\bar{E}_{K}+\bar{E}_{S}+\bar{E}_{P}+\bar{W}+2 \bar{C}=1
$$

In Eq. (27) the following definitions are used for the time averaged energy quantities, 
$\bar{E}_{\mathrm{x}}=\frac{1}{E_{0} T} \int_{0}^{T} E_{\mathrm{x}}(t) d t$, where subscript $\mathrm{x}$ is interchanged to denote the kinetic, strain and potential energies in the respective subregions. Additionally, $\bar{W}=\frac{1}{E_{0} T} \int_{0}^{T} W(t) d t$ and $\bar{C}=\frac{1}{E_{0} T} \int_{0}^{T} C(t) d t$

\subsection{Finite Element formulation}

For the numerical solution of the equivalent variational problem (Eq. 15), domain $\Omega$ is discretized and the unknown fields are approximated by means of the higher order finite element scheme developed in [17]. The discrete approximate solutions of the variational problem are given as,

$$
\eta^{h}=\sum_{i=1}^{6} H_{i}(x) \eta_{i}^{h}(t) \quad \text { and } \quad \varphi_{j}^{h}=\sum_{i=1}^{5} L_{i}(x) \varphi_{i j}^{h}(t), j=1,2 .
$$

Substituting the above into the discretized variation problem defined by Eq. (15) results in a second order system of the form $\mathbf{M} \partial_{t t} u+\mathbf{C} \partial_{t} u+\mathbf{K} u=0$, where vector $u$ contains the total nodal unknowns. Subsequently, a Newmark time integration scheme (see [17]) is employed in order to calculate the solution.

\section{Numerical Results}

In this section, a series of numerical results are presented using the physical parameters employed in the experiments described in $\mathrm{Wu}$ et al [32]. In the aforementioned work the length of the strip model was $L=10 \mathrm{~m}$, its thickness $\tau=0.038 \mathrm{~m}$ and the material elastic modulus $E=103 \mathrm{MPa}$. Moreover, the strip material density was $\rho_{p}=220 \mathrm{~kg} / \mathrm{m}^{3}$, and thus, its draft amounted to $d=0.084 \mathrm{~m}$ The experiment was performed in water depth of $1.1 \mathrm{~m}$, using incident wave heights of 5,10 and $20 \mathrm{~mm}$ and wave periods ranging from 0.5 to $3 \mathrm{~s}$, corresponding to deep and intermediate water depth conditions, respectively.

In order to comply with the shallow water assumption in the present work, the above physical data are used for calculations with a reduced water depth of $h=0.25 \mathrm{~m}$ (in non-dimensional terms $h=0.025)$, as shown in Fig. 2(1). Moreover, in order to illustrate the effects of variable bathymetry, two additional depth profiles, shown in 


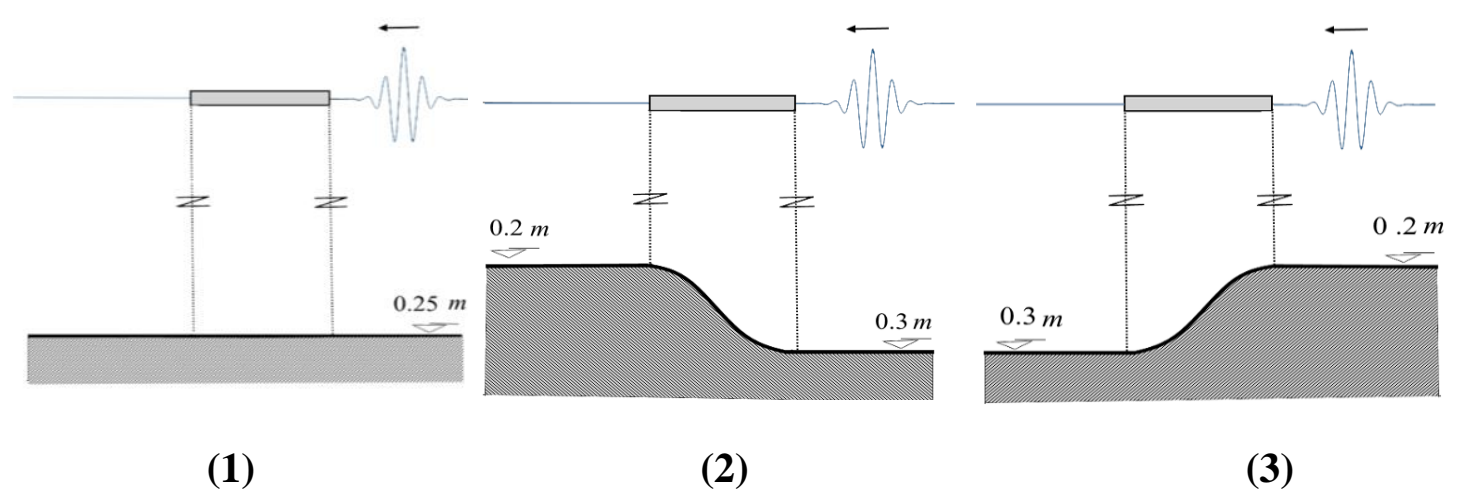

Figure 2 (1) Flat bottom profile. (2) Upslope and (3) downslope bathymetric profiles, with a mean bottom slope of $1 \%$.

Fig. 2(2) and (3) have been considered, corresponding to an upslope and a downslope environment with a mean bottom slope of $1 \%$. For the excitation $S(x)$ an incident wavepacket, with central wavelength $\lambda_{0}=4.5 \mathrm{~m}$ (in non-dimensional terms $\lambda_{0}=4.5$ ) and small amplitude $A=0.0076 \mathrm{~m}$, was considered in the following analysis. The imposed upper surface disturbance is described by,

$\eta(x, 0)=A f_{R}\left(x-x_{0} ; R\right) \cos \left(2 \pi x / \lambda_{0}\right)$,

where $f_{R}$ is a symmetric envelope of bandwidth $R$ with respect to $x_{0}$, which is the initial position of the wavepacket.

In the following section, Sec.4.1, a validation of the proposed methodology will be presented by comparing it against the analytical solution for the time harmonic responses of an elastic, floating structure. Comparisons are made for a strip employing an elastic connector at the upwave end of the structure and floating over constant shallow depth. Next, the effect of multiple connectors on the elastic responses will be studied in the time domain. Both a constant depth (Sec.4.2) and two mildly sloped bottom environments (Sec.4.3) will be considered.

\subsection{Validation against analytic solution for harmonic responses in constant depth}

For the case of thin, floating, elastic structures, in shallow water conditions and constant depth, the following 'shallow-wave equation of a freely floating board' derived by Stoker [33, Sec. 10.13, Eq. 10.13.74],

$$
K B^{2} \frac{d^{6} \psi(x)}{d x^{6}}+\left(1-M \omega^{2}\right) B^{2} \frac{d^{2} \psi(x)}{d x^{2}}+\mu \psi(x)=0,
$$


$\eta(x)=-\frac{i B}{\omega} \frac{d^{2} \psi(x)}{d x^{2}}$

is used. The above model refers to the linear harmonic responses of the structure. In the above expressions $\mu=\omega^{2} B$ is the frequency parameter. The non-dimensional frequency $\omega=\Omega \sqrt{L / g}$ is used, where $\Omega$ is the angular frequency. Variables $\psi(x), \eta(x)$ denote the complex amplitudes of the potential and the flexural deflection in the middle region $S_{0}$,

$\varphi_{0}(x, t)=\operatorname{Re}(\psi(x) \exp (-i \omega t)), \quad \eta(x, t)=\operatorname{Re}(\eta(x) \exp (-i \omega t))$.

The dispersion relation of Eq. (29a) is

$\mu B=K \kappa_{n}^{6} B^{2}+\left(1-M \omega^{2}\right) \kappa_{n}^{2} B^{2}$,

and its roots $\left\{ \pm \kappa_{n}, n=0,1,2\right\}$, the hydroelastic wavenumbers, are symmetrically distributed on the complex plane. The first root $\kappa_{0}$, is real and positive while roots $\kappa_{1}, \kappa_{2}$ have opposite real parts and equal positive imaginary parts. The solution of Eqs. (29) a-b is given by (see also Belibassakis \& Athanassoulis [20, Sec5.3 ]):

$$
\varphi(x)=\sum_{n=0}^{n=2} \alpha_{n} \exp \left(i \kappa_{n} x\right)+\beta_{n} \exp \left(-i \kappa_{n} x\right) .
$$

Similarly, in the free water surface subregions $S_{i}, i=1,2$, the harmonic solution of Eqs. (3) and (4) is given by

$\psi_{1}(x)=\mathrm{T} \exp \left(-i \kappa_{1}^{w} x\right), \quad \psi_{2}(x)=\exp \left(-i \kappa_{2}^{w} x\right)+R \exp \left(i \kappa_{2}^{w} x\right)$,

where $\psi_{i}(x)$ denote the corresponding complex wave potentials, $\mathrm{T}$ is the transmission coefficient of waves in $S_{1}$ and $R$ is the reflection coefficient of waves backscattered in $S_{2}$, respectively. The wavenumbers $\kappa_{i}^{w}$ in the water subregions $S_{i}$ $, i=1,2$ are provided by the asymptotic form of the water-wave dispersion relation in shallow conditions

$k_{i}=\sqrt{\mu_{i}} / B_{i}, \quad i=1,2$.

Finally, the coefficients $\alpha_{n}, \beta_{n}$ of Eq.(32), are easily determined from the boundary conditions Eqs. (12-14), at $x=0$ and $x=1$. These boundary conditions are expressed in terms of $\psi(x)$ through Eq. (29b), in conjunction with the following end conditions 


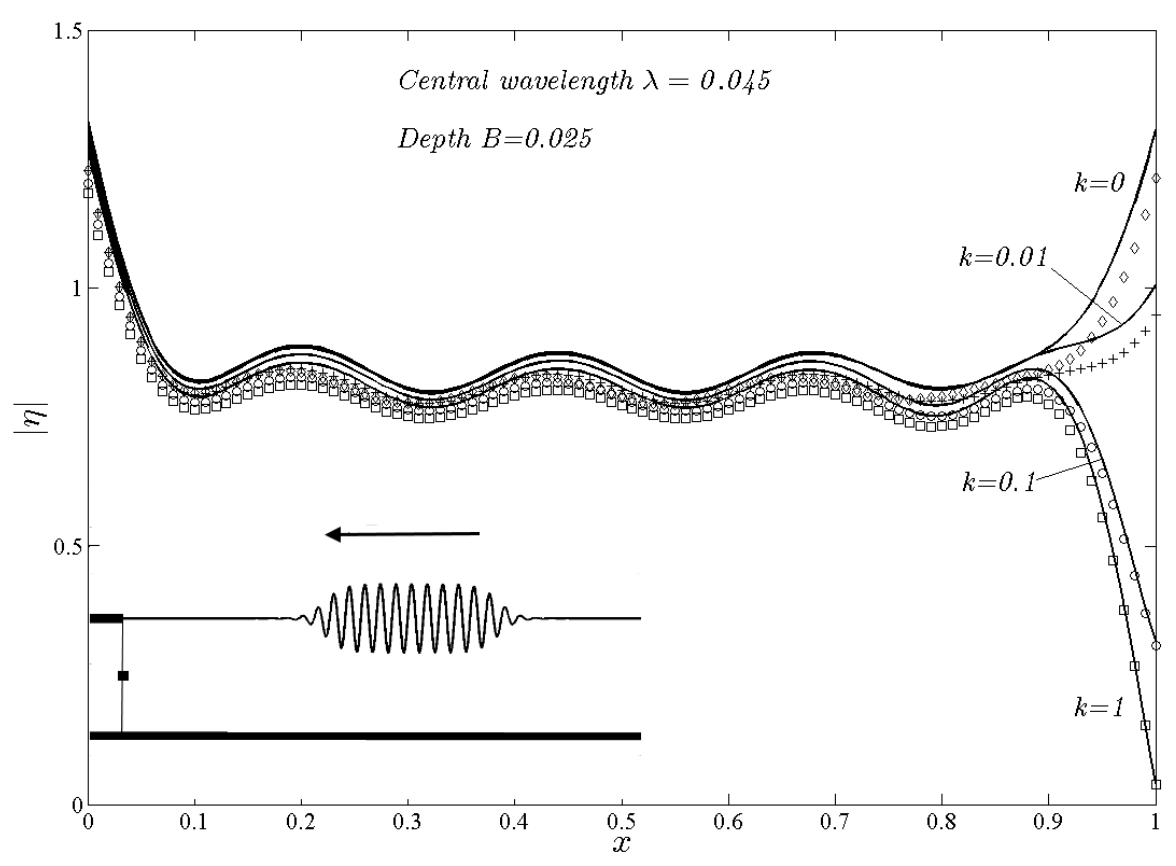

Figure 3 Harmonic responses of a thin elastic plate in constant depth and shallow water conditions, with an elastic connector of stiffness $k$ located at $x=1$. The analytical solution is represented by solid lines, while the FEM solution is denoted by diamonds $(k=0)$, stars $(k=0.01)$, circles $(k=0.1)$, and squares $(k=1)$.

$$
\frac{d \psi}{d x}+i \kappa_{1}^{w} \psi=0, \text { at } x=0, \text { and } \frac{d \psi}{d x}-i \kappa_{2}^{w} \psi=-2 i \kappa_{2}^{w} \exp \left(-i \kappa_{2}^{w}\right) \text {, at } x=1 \text {. }
$$

They provide the matching of the complex wave potential $\psi(x)$ at the interfaces between the tree subregions.

In order to calculate the harmonic responses of the hydroelastic system by the proposed time-domain method a very broad ramp function $f_{R}$, containing a multiple number of wavelengths, is used. A comparison against the analytical solution is presented in Fig. 3 for a frequency parameter $\mu=0.117$, corresponding to depth $B=0.025$ and central wavelength $\lambda=0.45 \mathrm{~m}$ (non-dimensional $\lambda=0.045$ ), and thus ensuring shallow wave conditions.

More specifically, in Fig. 3 the harmonic responses of the freely floating board with an elastic connector located at its right end, at $x=1$, are shown. Results are calculated by the analytical solution of Stoker's model and by the presented FEM for a various connector stiffness coefficients $k$ and zero damping are plotted. These stiffness values include the freely floating case, corresponding to $k=0$, with 
increasing $k=0.01,0.1$, and 1 as shown in the figure. The proposed method solutions are found to be in good agreement with the analytical solution, for all values of the examined connector stiffness. The small deviations are attributed to the approximation of the harmonic response of the structure by means of the presented transient methodology.

Furthermore, in Fig. 3, it can be seen that for a very stiff connector $(k=1)$, the elastic deflection of the structure at the upwave connected end almost vanishes. The above fact leads to the conclusion that the wave induced vibration of the elastic structure, in the vicinity of the elastic connector, becomes weaker (and eventually vanishes) with increasing connector stiffness. We note here that this finding is in contradiction with the results reported by Cunbao et al (2007, Figs.13-15), although the latter studies are not directly comparable since they refer to intermediate and deep water conditions.

\subsection{Constant depth environment}

The constant depth profile, illustrated in Fig 2(1) is initially examined. The horizontal domain is appropriately truncated, and the present system is integrated up to the time ensuring that no reflections from the computational domain boundaries are backscattered, contaminating the numerical solution $(T=76)$. For the calculation of the plate response, 200 hydroelastic elements were employed, along with 8000 timesteps. Initially, the freely floating strip response is examined. A series of snapshots, showing the propagation of the initial disturbance, is presented in Fig. 4, for the freely floating case, i.e. $k_{n}=c_{n}=0, n=1,2, \ldots, N+1$. For illustration purposes the non-dimensional upper surface elevation is plotted ten times larger in the given figure. The initial excitation (Eq. 28) with $f_{R}(x)=\exp \left(\alpha\left(x-x_{0}\right)^{2}\right)$, where $\alpha=11.5$ and $x_{0}=9.3$, modeling a narrow band pulse, is used in the calculations. The pulse is split into two waveforms traveling in opposite directions at constant speed (Fig. 4b). As the two waveforms are not dispersive, their forms remain unaltered while traversing the water region $S_{2}$. In Fig. 4(c) the waveform propagating towards the negative $x$ axis, is seen to approach the free edge of the elastic strip at $x=1$. Subsequently, after wave impact, the propagation of the hydroelastic pulse is plotted in Figs. 4(d)-4(h). The incident wave is partially reflected, backpropagating in the right subregion $S_{2}$, 

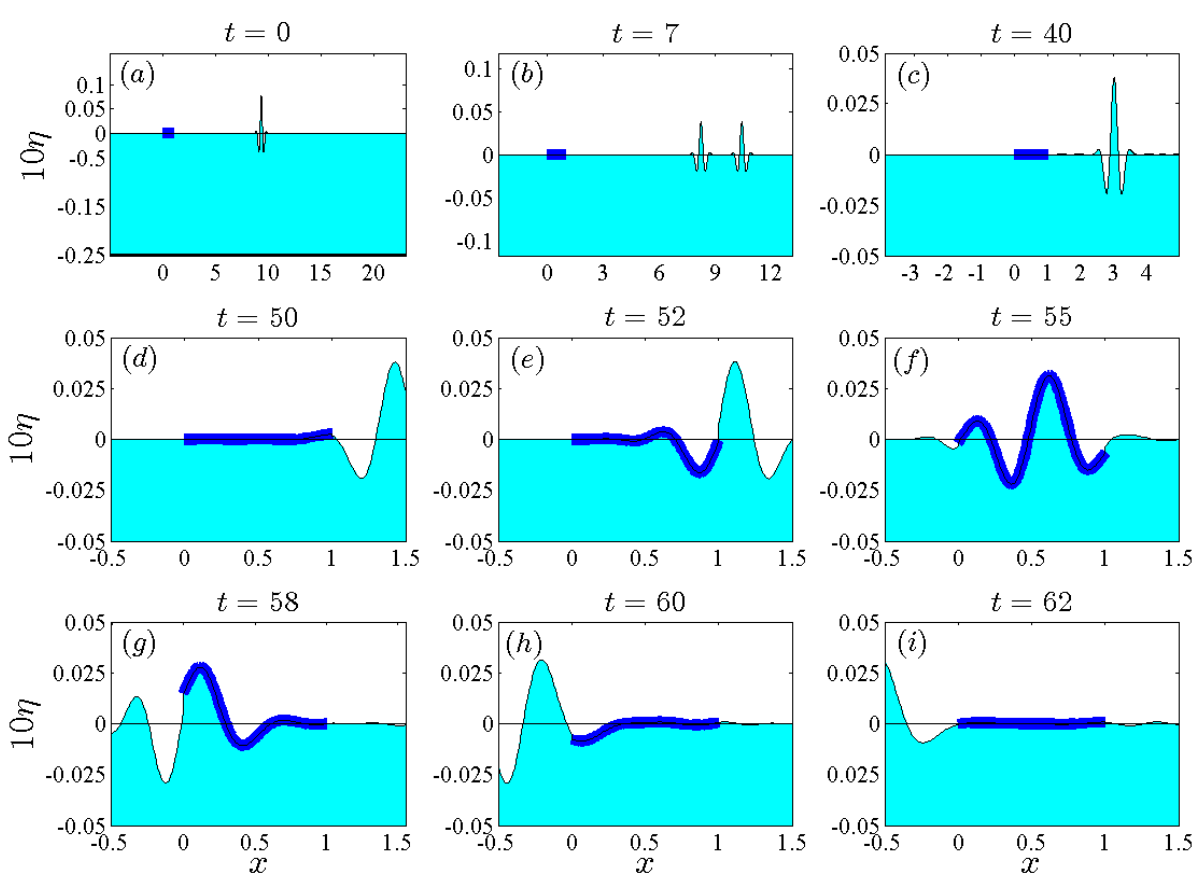

Figure 4 Snapshots of the wavepacket propagation in domain $\Omega$ for the case of a constant depth profile (1). Note that for illustration purposes the non-dimensional free-surface elevation and the plate deflection are multiplied by 10 .

and partially transmitted in the left subregion $S_{1}$, as seen in Fig. 4(h). The structure eventually approaches a state of rest in Fig. 4(i). Next, the effect of the employed elastic connectors on the hydroelastic response of the strip is investigated for the same environment and incident wave. In the following analysis two and three elastic connector-strip configurations with $k_{n}=k, c_{n}=c$, where $n=1,2$ for the former case and $n=1,2,3$ for the later, are considered. In Fig. 5 the deflection of a strip featuring two elastic connectors positioned at the free ends ( $x=0$ and $x=1)$, is plotted for an extended range of characteristic non-dimensional stiffness values $k=\left\{\begin{array}{lll}1 & 0.1 & 0.01\end{array}\right\}$, and zero damping, i.e. $c=0$. Calculated results are compared against the freely floating case response. The deflection of the elastic strip for different elastic connector stiffness values is shown at three distinct instances in time, representing the phases of wave entry in the middle subregion $S_{0}$, the hydroelastic pulse propagation and the transmission into the downwave subregion $S_{1}$. The non-dimensional bending moment distributions along the elastic strip are also presented for the same time instances. 

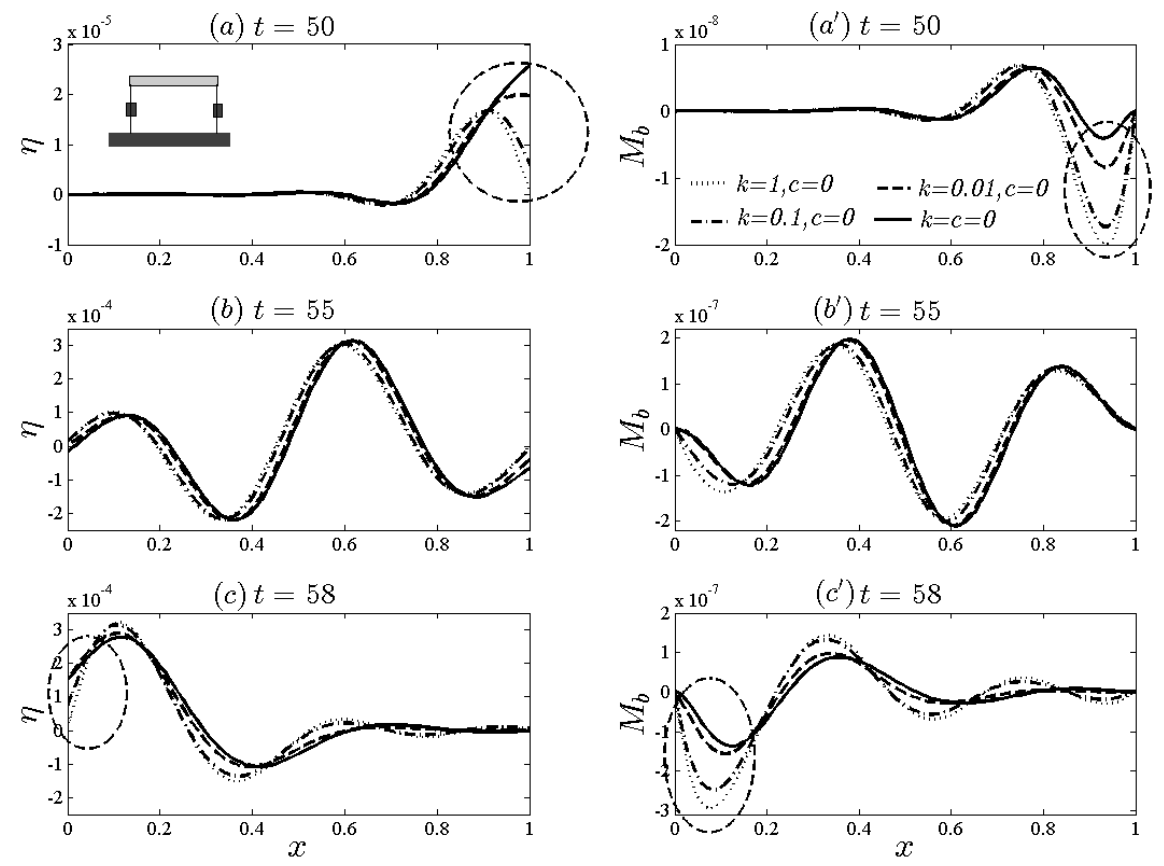

Figure 5 Non-dimensional strip deflection (left subplots) and bending moment $M_{b}=K \eta_{x x}$, (right subplots) distribution for several connector stiffness values and zero damping. Two connector configuration for bathymetric profile (1).
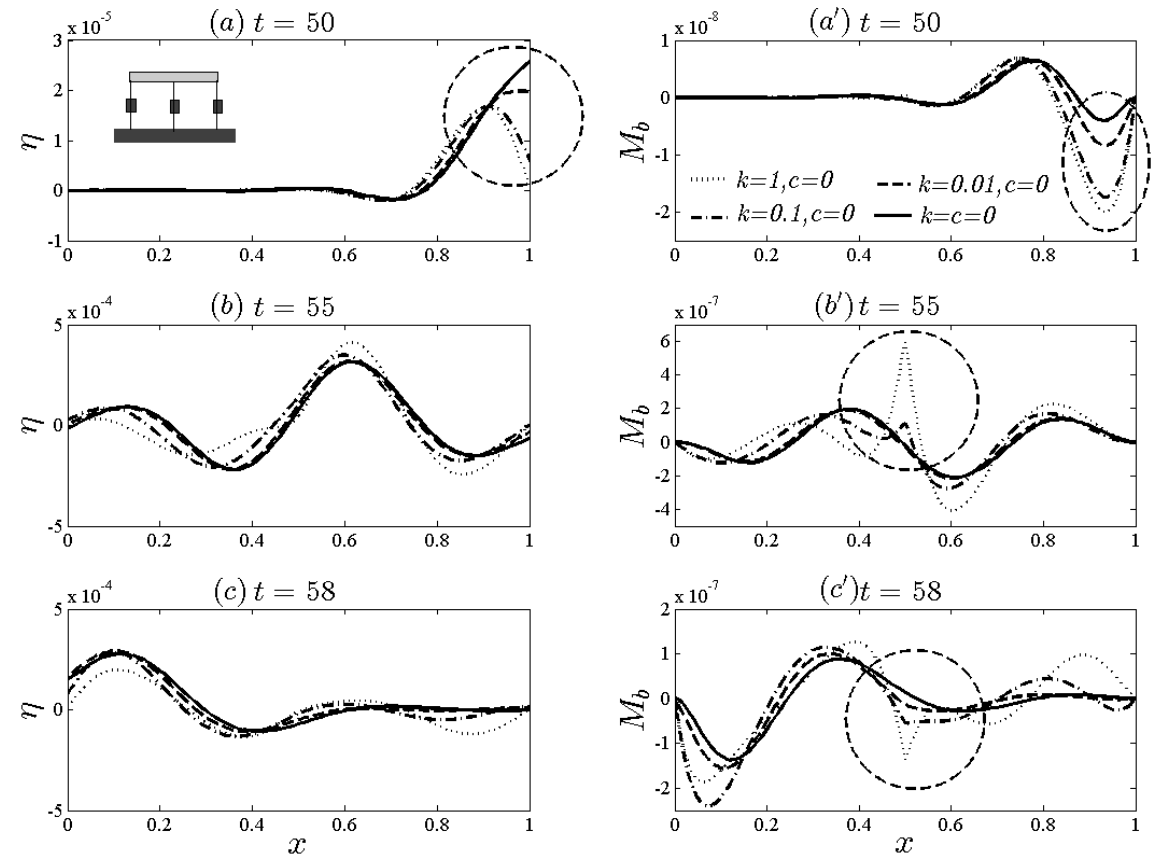

Figure 6 Same as Fig. 5 for the three connector-strip configuration. 
It is observed that during the wave entry phase, increasing the connector stiffness, reduces the deflection, and increases the bending moment values in the vicinity of the strip end $(x=1)$, as indicated by the dashed areas in the Figs. 5(a) and 5(a'). Compared to the freely floating response at the given moment in time $(t=50)$, setting $k=0.01,0.1$ and $k=1$ reduces the maximum absolute strip deflection by $22.8 \%$, $34.8 \%$ and $35.5 \%$, respectively. On the other hand, the calculated maximum absolute bending moments substantially increase with increasing connector stiffness, at the vicinity of the free edge, reaching an intensification of over $200 \%$ for $k=1$. This can be attributed to the local restriction imposed on the elastic motion of the strip by the connector at $x=1$. Next, in the hydroelastic pulse propagation phase, examined in Figs. 5(b) and 5(b'), the maximum deflection reductions achieved by the employment of the edge connectors reaches $0.25 \%, 3 \%$ and $4.85 \%$ for $k=0.01,0.1$ and $k=1$ respectively. The calculated, maximum bending moment at $t=55$, also appears reduced by $0.4 \%, 5 \%$ and $8.2 \%$ for the corresponding stiffness coefficient values.

During the wave transmission phase, increasing connector stiffness results in larger moduli of deflections and bending moments, in the vicinity of the downwave end of the structure, as indicated by the dashed areas in Figs 5(c) and 5( $\left.\mathrm{c}^{\prime}\right)$. Particularly, for $k=0.01,0.1$ and $k=1$ maximum absolute deflection increases by $4.31 \%, 13.45 \%$ and $15.9 \%$, respectively.

The imposed restriction on strip deflection is magnified with increasing connector stiffness, causing the flexural response of the strip to intensify locally at the strip edges during wave impact and hydroelastic pulse transmission. The latter has a profound effect on both the flexural deflection of the structure and the induced bending moment profiles. Examining the overall responses in time, the maximum absolute deflection was significantly reduced (by $29.26 \%$ ) for $k=0.1$ while the maximum absolute bending moment of the elastic strip is increased by $35.4 \%$, compared to the freely floating case. The overall maximum absolute deflection was also effectively mitigated by setting $k=0.01$ (22.36\%) and $k=1$ (27.49\%). However, increasing connector stiffness led to magnification of the maximum absolute bending moment, by $0.21 \%, 35.4 \%$ and $62.47 \%$ for increasing stiffness coefficients. The previous observation suggests that deflection mitigation through connector stiffening might lead to undesirable, excessive stresses due to flexural motion.

In Fig. 6 a system with three elastic connectors is examined. The previous configuration is enhanced by a third connector, positioned at the middle of the elastic 
strip ( $x=0.5)$. At wave entry, shown in Fig. 6(a), the deflection appears to be reduced by $35.5 \%$ for $k=1$, compared to the freely floating case, while bending moment intensification is observed in Fig. 6(a') in the vicinity of the strip upwave edge (depicted once again by the dashed area). In Fig. 6(b) (at $t=55$ ) the strip deflection, once again compared with the freely floating case, increases by $1.1 \%$, $11 \%$, and $30.1 \%$, for $k=0.01,0.1$ and $k=1$, respectively. At the same instance, the calculated maximum absolute bending moment also appears to be magnified, as shown in 6(b'). This is attributed to the overstiffening of the system due to the presence of the middle elastic connector. The kink in bending moment distribution observed in Fig. 6(b') for $k=1$, at the middle of the floating strip, is indicative of the induced, excessive local stresses due to bending, attributed to the imposed restriction on deflection. At $t=58$, (Fig. 6c) the deflection almost vanishes for $k=1$, showing a reduction of $29 \%$ compared to the freely floating case. Maximum overall deflection reduction (over time) is achieved for $k=0.01$ (by $22.32 \%$ ) when compared with the free strip response. On the other hand, overall maximum, absolute bending moment is increased by $180 \%, 35 \%$ and $2 \%$ for stiffness coefficients $k=1,0.1$ and $k=0.01$. Hence it is deduced that the intensity of flexural effects, i.e. induced maximum bending moment values, rise with increasing connector stiffness for both examined configurations when compared with the freely floating case.

Next, the combined stiffness and damping effects of the elastic connectors on the hydroelastic response of the studied system, in constant depth, are studied. To this purpose, the resulting maximum absolute deflection and maximum absolute bending moment values are calculated. The same set of damping coefficients $\mathrm{c}=\{1,0.1,0.01,0\}$ and an extended interval of stiffness coefficient values $0<k<10$ are used. Notably, the above interval selection includes the values of interest for practical applications. In Figs. 7 and 8 the maximum absolute deflection and the maximum absolute bending moment distributions are presented for both examined configurations featuring two and three connectors. As expected, the calculated maximum absolute deflection corresponds to the undamped case, i.e. $c=0$, and small stiffness coefficient values (Figs.7 a, b). 

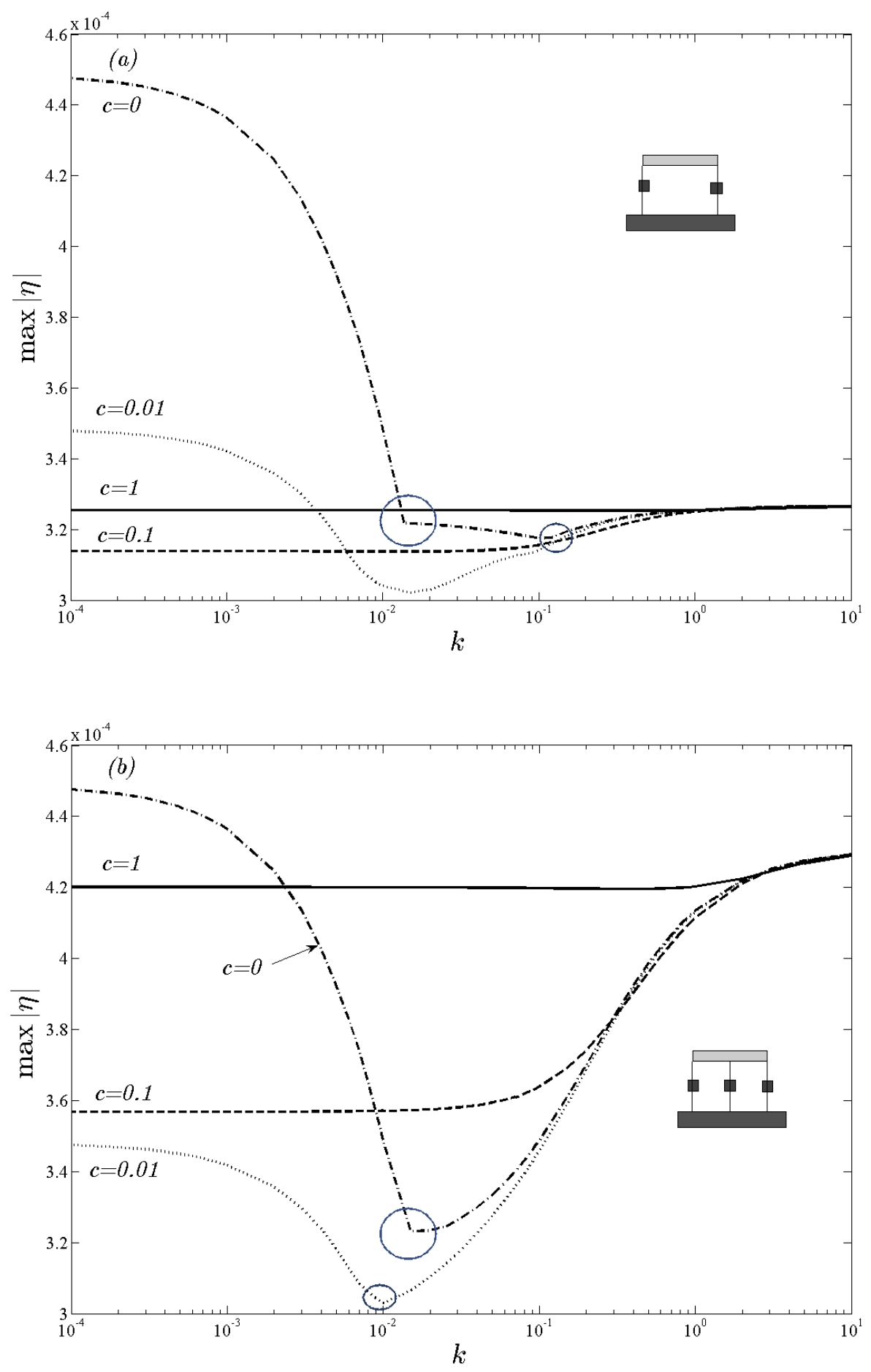

Figure 7 Semi-log plot of the maximum absolute deflection: (a) two edge connectors, (b) three connectors.

It is noted that for large stiffness coefficient values, the maximum absolute deflection is practically independent of the studied damping parameter values. Additionally, it is observed in Fig.7 (a) and (b), that the optimal damping parameter, minimizing the maximum absolute deflection, is generally dependent on the stiffness of the 
connectors. In the overdamped case $(c=1)$ the above correlation appears weaker. Thus, it is deduced that it is possible to achieve minimization of the flexural deflections of a given configuration, for certain stiffness and damping coefficients ( $c=0.01$ and $k \sim 10^{-2}$ in the considered examples), by means of the proposed methodology.

For the same example, in Fig. 8, it is shown that the maximum (absolute) bending moment, calculated for the three-connector configuration ( Fig. 8b) is larger than the obtained value for the two-connector strip configuration, examined in Fig. 8 (a). The above is attributed to the system overstiffening due to the presence of the added middle connector.

Furthermore, as the stiffness coefficients $k$, become very large, the maximum calculated (absolute) bending moment seizes to depend on the damping coefficient. This phenomenon is illustrated by the plateau areas depicted in both Fig. 8 (a) and (b). Finally, the observable points of inflection in Figs. 7 and 8, noted by the circled areas, are associated with abrupt changes in the location of the maxima values along the strip.

In order to gain a better understanding of the energy exchange between subregions, during the hydroelastic pulse excitation and propagation, the various terms composing the total energy of the system are studied. An illustration of the energy balance, expressed by Eq. (19), is shown in Fig. 9 for the case of a two-connector configuration, with $k=c=0.01$. The total energy of the system, including the dissipated energy due to connector damping effects, is denoted by the solid black line, and remains constant in time. The energy of the water column in subdomain $S_{2}$ decreases after the moment of wave impact. After the excitation of the floating strip, the hydroelastic pulse begins to propagate in the middle region. Concurrently, the sum of the strain, kinetic and potential energy of the strip increases until a state of rest is reached and the quantities vanish after the full transmission of the pulse into the left halfstrip. Although the elastic connector energy $W(t)$ vanishes, the dissipated energy due to connector damping, represented by quantity $C(t)$, remains constant in time after the strip reaches a state of rest once again. Hence, the total connector energy $W(t)+2 C(t)$, increases after wave impact and remains constant after wave transmission into $S_{1}$. Finally, as the wave train enters the left half strip, the sum of 
Published in Marine Structures, 48, 33-51. doi:10.1016/j.marstruc.2016.04.002
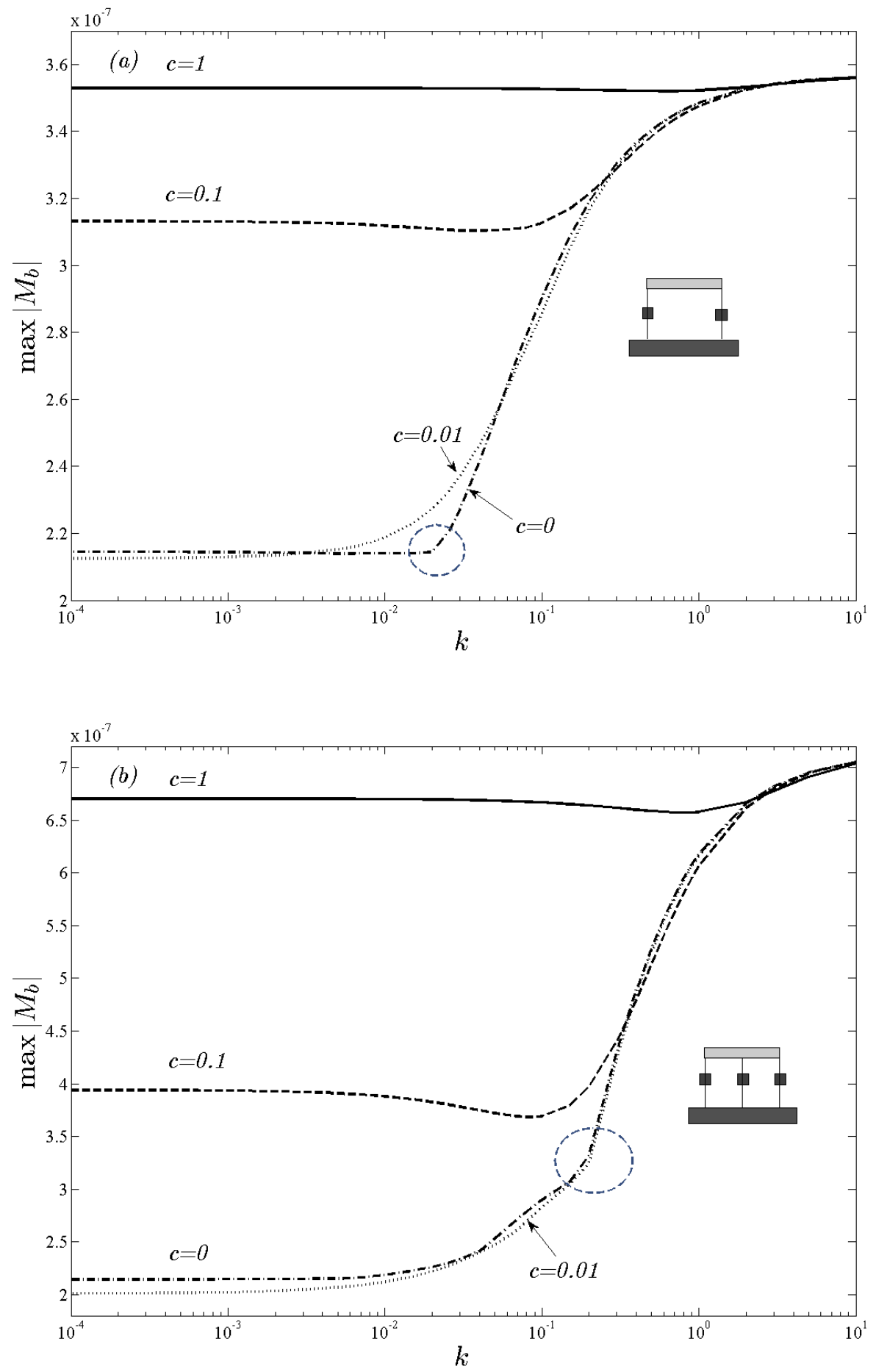

Figure 8 Semi-log plot of the maximum absolute bending moment: (a) two edge connectors, (b) three connectors. 


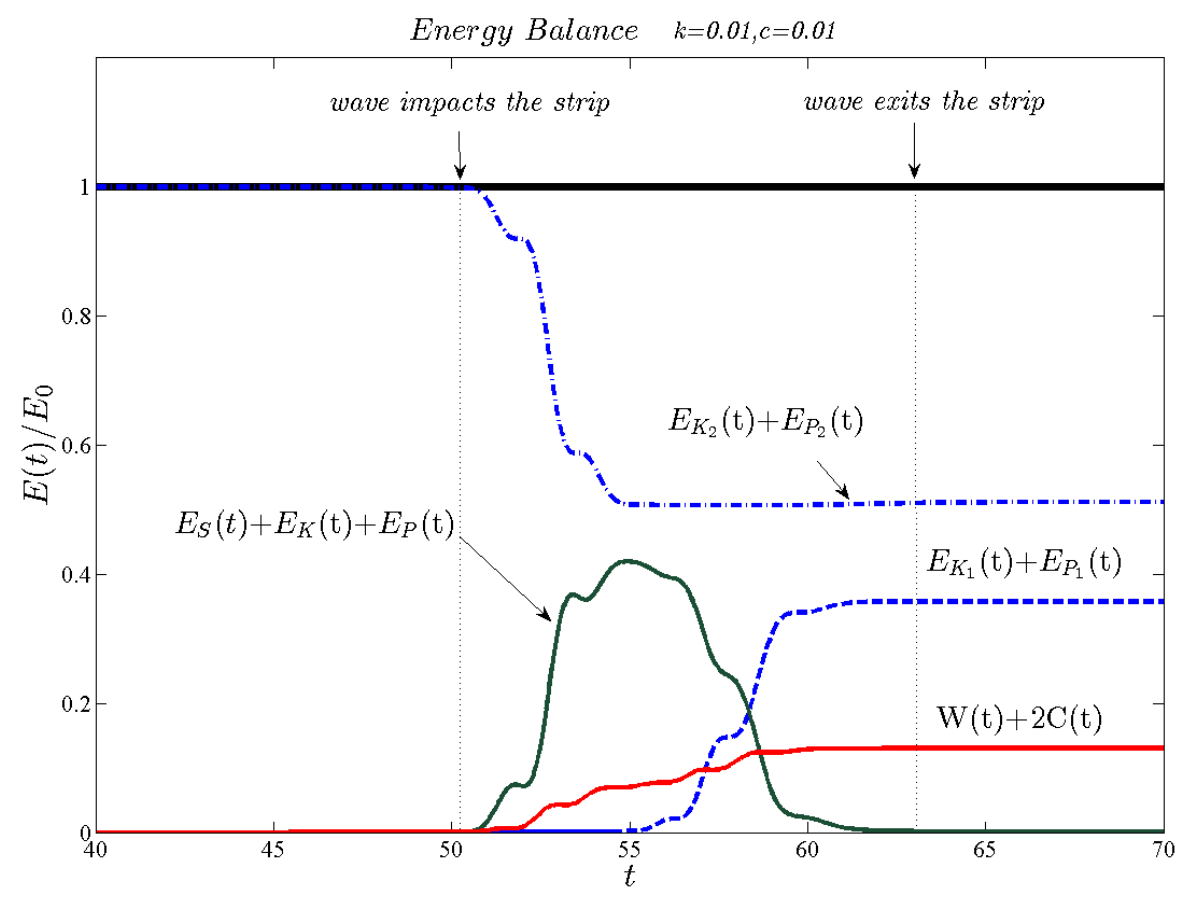

Figure 9 The energy balance for a two connector configuration with $k=c=0.01$

the kinetic and potential energy of the water column in this region increases until full wave transmission in $S_{2}$ is achieved.

Next, a correlation between the energy quantities, defined in Sect. 3.1, and the elastic connector parameters is examined for the studied thin, elastic strip, employing two and three connectors and floating over the constant depth profile (1). It is noted here that the minimisation of the strip kinetic energy is particularly interesting for the design of hydroelastic response mitigating devices and systems. In addition, structural safety and robust design would be translated in strip strain energy minimisation, while efficient wave energy harvest into dissipative energy maximisation. To this aim, the correlation between the energy quantities and the elastic connector parameters is further investigated in Figs. 10 and 11, for the defined strip-connector configurations. In Figs 10(a) and 11(a) the elastic spring energy averaged in time, $\bar{W}$ is examined for a range of spring coefficient values. As expected, when the connector stiffness is small, less elastic energy is stored, while on the other hand, as the system is over stiffened the strip deflection is restricted, resulting again in smaller potential energy sums. Additionally, the elastic spring energy is found to increase with decreasing damping parameters in both cases. Naturally, increasing the damping parameter 

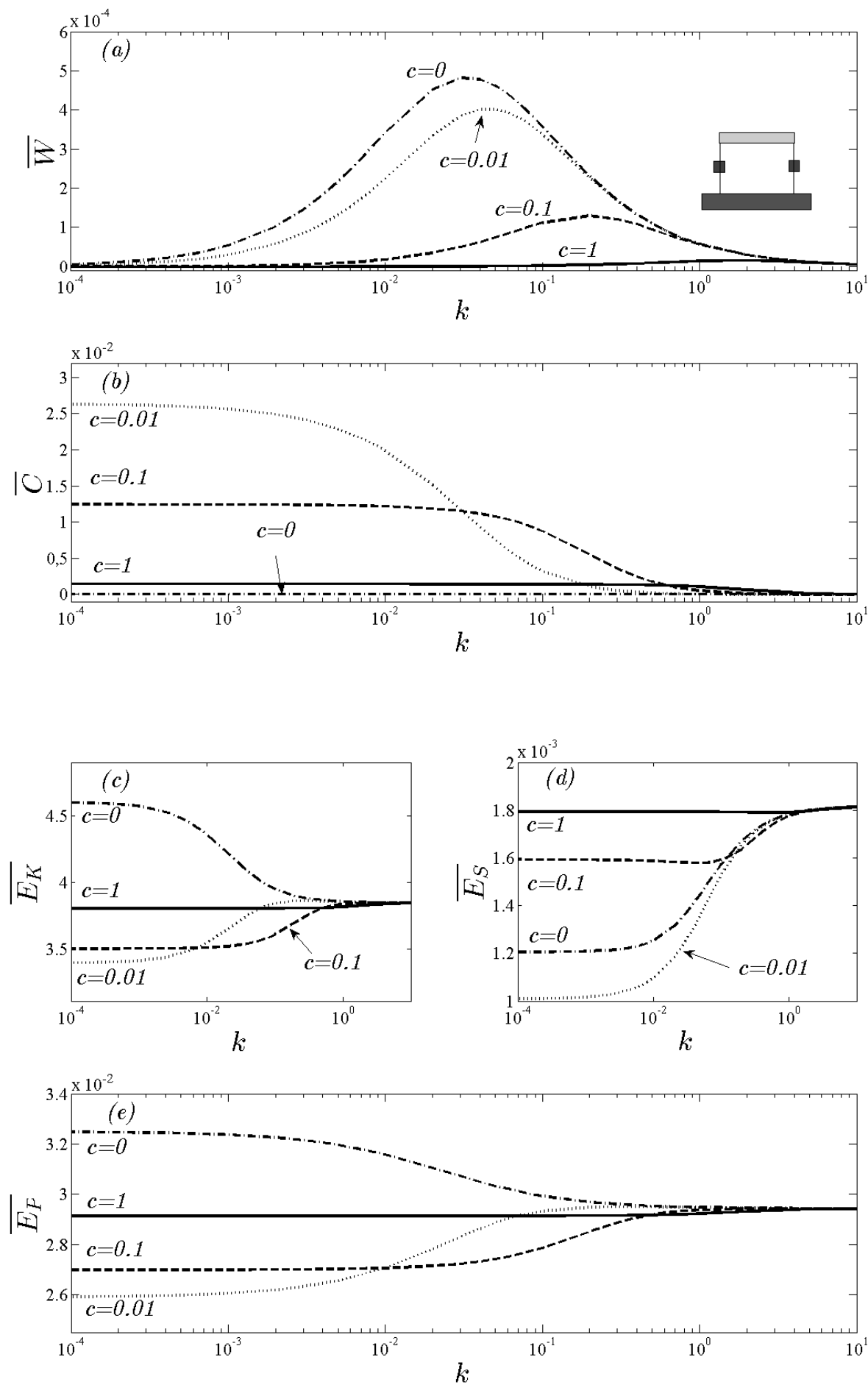

Figure 10 Semi-log plot of the averaged energy quantities for the two-connector case.

Connector parameters (a) elastic $\bar{W}$ and (b) damping $\bar{C}$. Elastic strip energy parameters (c) kinetic energy $\bar{E}_{K}$, (d) strain energy $\bar{E}_{S}$ and (e) potential energy $\bar{E}_{P}$. 

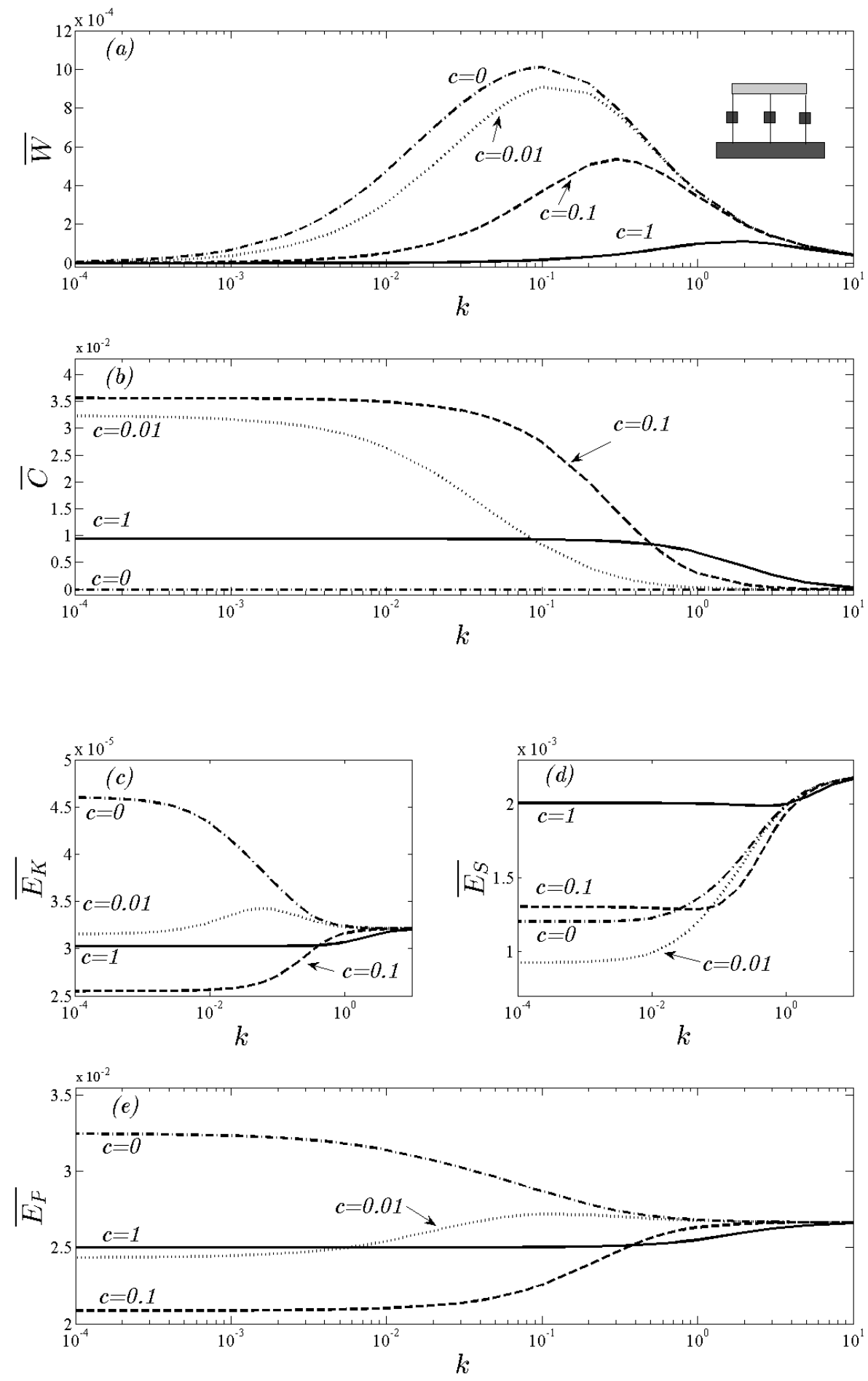

Figure 11 Same as Fig 10 for the three connector-strip configuration.

results in a larger restoring force term which minimizes deflection. Finally, the near resonance conditions concerning the entire system for a given elastic strip are dependent on both connector stiffness and damping coefficients and is clearly depicted by the maxima of the $\bar{W}$-curves concerning the elastic connector energy. The damping energy is associated with the oscillatory speed of the strip (see Eq. 25). 
In both cases, featuring two and three connectors, overstiffening results in vanishing damping energy, regardless of the damping parameter. The previous fact is straightforward, since in the presented 1-D hydroelastic system, the intensification of the restoring force on the strip results in energy reflection back in the free surface region. This fact essentially leads to less energy sums being transmitted into the middle subregion $S_{0}$. Additionally, it is observed in Figs 10(b) and 11(b) that for a given configuration and connector stiffness parameter, there exist specific values of the damping coefficient for which dissipated energy is maximized. This is expected to have an important effect on the kinetic energy of the elastic strip $\bar{E}_{K}$. The kinetic energy is presented in 10 (c) and 11(c) for a combination of stiffness and damping coefficients for the two and three connector configuration, respectively. Maximum kinetic energy is obtained when the restoring force is minimal, hence for $c=0$ and $k \sim 10^{-4}$. Since the kinetic energy of the strip is also a function of oscillatory motion speed (see Eq. 22a), minimization is achieved for the damping parameter values maximizing energy dissipation, as previously described.

The averaged strain energy $\bar{E}_{S}$, which expresses the amount of flexural deformation undergone by the strip (defined by Eq.29b), is examined in Figs 10(d) and 11(d). It is observed that in both cases the minimum strain energy is obtained for damping coefficient value $c=0.01$. This is expected since the maximum absolute bending moment, as seen in Figs. 7(a) and (b), was also minimized for $c=0.01$ in both examined strip-connector configurations. Notably, the strain energy of the strip, in both cases, becomes larger with increasing stiffness coefficients which correlates with previous observations for the bending moment, illustrated in Fig 8. Finally, the total energy $\bar{E}_{P}$ in the middle subregion is depicted in $10(\mathrm{e})$ and 11(e). Since the elastic strip deflection is generally very small, the energy sum expressed by $\bar{E}_{P}$ is dominated by the kinetic energy of the water column in the middle region $\left(S_{0}\right)$ and resembles the kinetic strip energy plotted in Figs. 10(c) and 11(c).

\subsection{Sloping Bottom Profiles (b) and (c)}

Variable bathymetric effects, as previously mentioned, are an important consideration in nearshore and coastal marine structure design. The proposed computational tool, able to account for a variable seabed, could be found useful in the study of 
bathymetric effects on the hydroelastic response of a floating strip with elastic connectors. In this section, numerical results are presented and discussed for the two variable seabed profiles corresponding to an upslope (b) and a downslope (c) environment (see Fig 2).

More specifically, in Figs. 12 and 13, the strip responses and bending moment distributions for the two connector-strip configuration are plotted at three distinct time instances for profiles (b) and (c) respectively. Curves corresponding to various connector stiffness parameter values are presented, while zero damping effects were considered. In accordance with previous observations, (see Fig. 5) it is established that increasing connector stiffness, results in larger maximum absolute bending moment values. The above leads to increased normal stresses induced by flexural motion but an overall reduced hydroelastic response compared to the freely floating case, for both profiles of variable bathymetry.

In the case of the upslope environment, it is observed in Fig. 12(a) that at the wave entry phase $t=48$, the maximum absolute strip deflection appears reduced by $19.24 \%$, $54.5 \%$ and $55 \%$ for $k=0.01,0.1$ and 0.1 respectively, compared to the freely floating case. Marginal response reduction is achieved at $t=50$ (Fig. 12b), reaching $0.12 \%, 1.95 \%$ and $3.34 \%$ for $k=0.01,0.1$ when compared to the freely floating case. At wave exit, the maximum absolute strip deflection is only slightly reduced by $1.1 \%$ for $k=0.01$ while it increases by $3.85 \%$ and $8 \%$ for $k=0.1$ and $k=1$. The overall (over time) maximum absolute deflection is reduced by $22.36 \% 29.45 \%$ and $28.23 \%$ for $k=0.01,0.1$ and 1. In Fig. 12(c) absolute maximum deflection increased by $3.85 \%$ and $8 \%$ for $k=0.1,1$, while marginal reduction of $1.19 \%$ is achieved for $k=0.01$. The above findings are in agreement with previous observations for the constant depth case (see Fig 5).

The corresponding bending moment distributions, presented in Fig 12 (a')-( $\left.\mathrm{c}^{\prime}\right)$, exhibit intensification of flexural effects in the vicinity of the strip edges during wave entry and exit (denoted by the dashed areas), which was also observed in the constant depth case. However, maximum absolute bending moment intensification is reduced compared to the constant depth profile calculations, reaching $48.2 \%$ and $93.7 \%$ for $k=0.1$ and 1 at wave entry, while a slight decrease of $0.39 \%$ compared to the freely floating case in bathymetric profile (2) is achieved for $k=0.01$. At wave propagation stage $t=50$, maximum absolute bending is slightly increased by $1.16 \%, 2.32 \%$ and $1.15 \%$ for increasing stiffness. This can be attributed to the fact that the propagating 

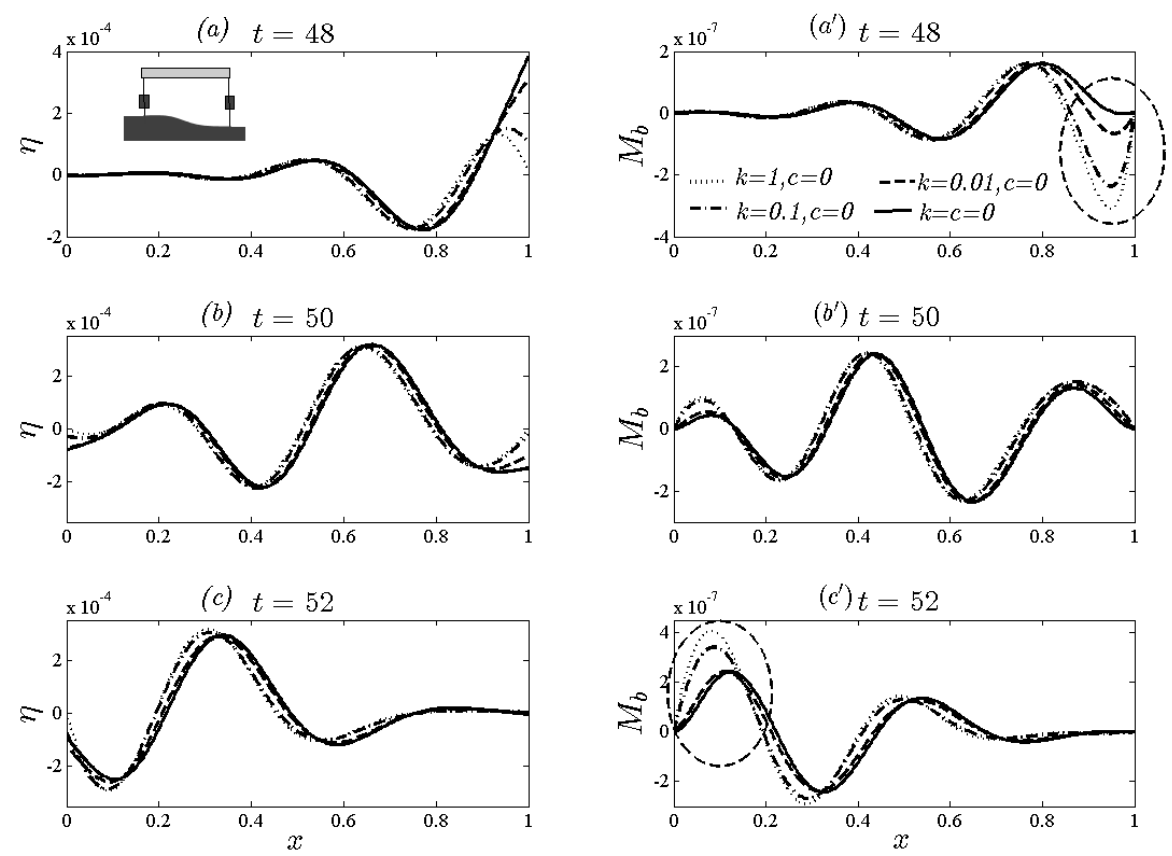

Figure 12 Non-dimensional strip deflection (left subplots) and bending moment $M_{b}=K \eta_{x x}$, (right subplots) distribution for several connector stiffness values and zero damping. Two connector-strip configuration for bathymetric profile (2).
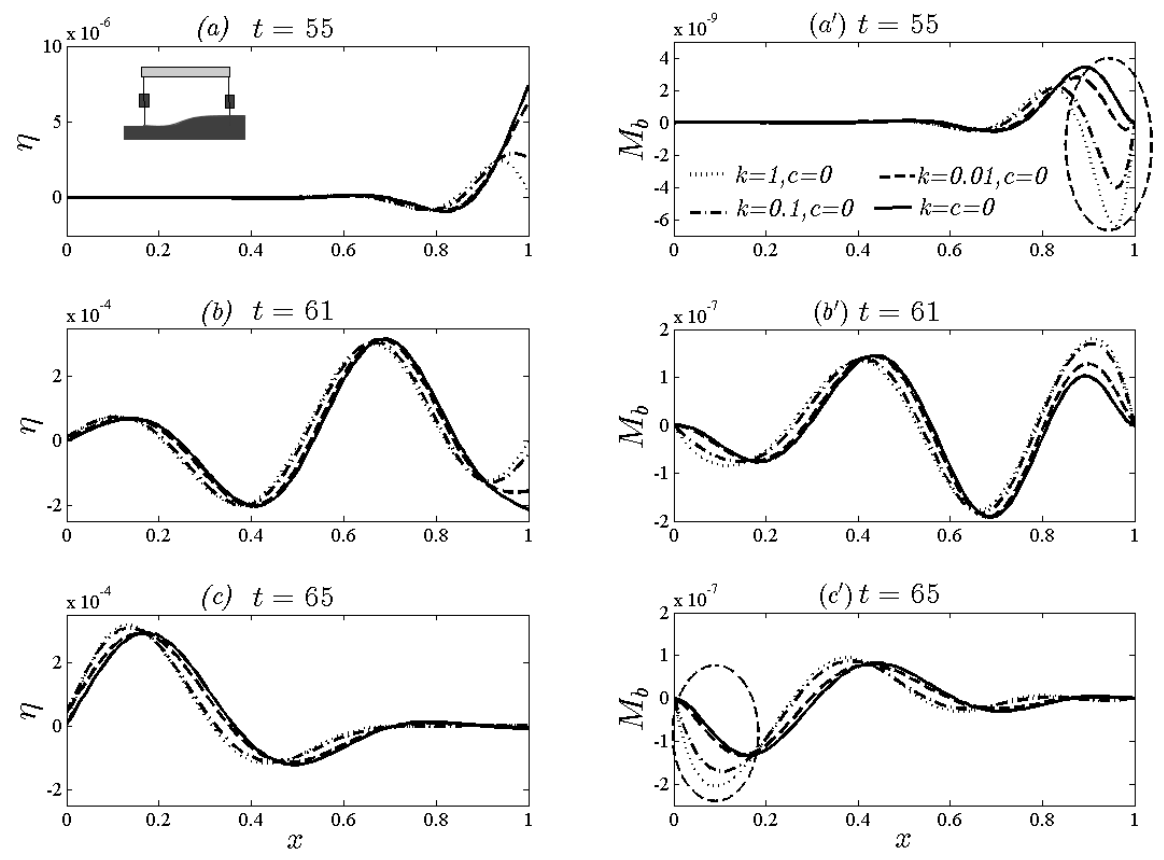

Figure 13 Same as in Fig.12 but for the downslope environment (3). 
pulse becomes steeper with decreasing depth (profile b), causing an intensification of flexural effects. During wave exit bending moment intensification is observed, with maximum increase reaching $66 \%$ for $k=1$ compared to the freely floating case.

Finally, the hydroelastic responses of the two connector-strip configuration floating over the downslope bathymetric profile (c) are examined in Fig. 13. Overall maximum strip deflection is once again reduced by $22.35 \%, 29.08$ and $27.68 \%$ for increasing connector stiffness values. Moreover, bending moment intensification is observed at the vicinity of employed connectors at wave entry and transmission. Hence, bathymetric effects appear to have minimal impact on the hydroelastic response of the examined configurations.

Increasing the connector damping parameter while keeping the stiffness value constant was also found to reduce the strip elastic motion. Examining the bending moment distributions for the varying damping analysis it was observed that bending moment is magnified in the vicinity of the free edges during wave entry and exit. This were the case for both considered profiles. Hence, the inclusion of dampers in the elastic connector design might have an undesirable intensification effect in the induced stresses on the strip. In conclusion, the design of an efficient elastic connector configuration constitutes a multi-parametric optimization problem. The proposed methodology is able to provide useful information concerning the vibration reduction of the structure and support the design of efficient mooring systems.

\section{Conclusions}

The time-domain hydroelastic response of a thin, floating strip, elastically connected to the seabed, is examined in the present work. Based on the variational formulation of the initial-boundary value problem in shallow water conditions, an energy balance equation is derived, while a higher-order finite element scheme is implemented for the numerical solution. Results for various strip-connector configurations of interest, illustrating the response reducing effects of the employed connectors, are presented. In addition to the flat bottom case, two variable bathymetric profiles (an upslope and a downslope environment) were studied. Numerical results were obtained for the cases of two strip-connector configurations. The first configuration employs two elastic connectors, positioned at the free strip ends, while the second features an additional connector located at the middle of the structure. The study of the aforementioned configurations reveals that response mitigation is possible through the increase of 
connector number and stiffness. However, deflection mitigation through connector stiffening is associated with excessive maximum bending moment values, at the vicinity of the connector locations along the strip. Hence, overstiffening can be correlated with undesirable bending induced local stresses. Moreover, optimal damping coefficient for the minimization of the maximum absolute defection and bending moment is found to be generally depended on connector stiffness. In conclusion, the design of an efficient elastic connector-strip configuration constitutes a multi-parametric optimization problem. The proposed methodology is able to provide useful information concerning the vibration reduction of the structure and support the design of efficient motion mitigating systems. Future research will focus on the treatment of the 3D problem and intermediate water depth effects. Finally, the investigation of weak nonlinearity is of equal importance. An initial investigation in the latter direction has been presented in Karperaki et al [35].

\section{Acknowledgements}

The present work has been supported by the project HYDELFS funded by the Operational Program "Education and Lifelong Learning" of the National Strategic Reference Framework (NSRF 2007-2013) -Research Funding Program ARHIMEDES-III: Investing in knowledge society through the European Social Fund. In particular, author Theodosios K. Papathanasiou acknowledges support from the aforementioned program for the period from 6/9/2012 to 30/09/2014.

\section{References}

[1] Wang CM, Watanabe E, Utsunomiya T. Very large floating structures. London, UK: Taylor and Francis; 2008.

[2] Watanabe E, Utsunomiya T, Wang CM. Hydroelastic analysis of pontoon-type VLFS: a literature survey. 2004, Eng Struct 2004; 26:245-256.

[3] Wang CM, Tay ZY. Very Large Floating Structures: Applications, Research and Development. Procedia Eng 2011; 14: 62-72.

[4] Chen XJ, Wu YS, Cui WC, Juncher Jensen, J. Review of hydroelasticity theories for global response of marine structures. Ocean Eng 2006; 33: 439-457.

[5] Ohmatsu S. Overview: research on wave loading and responses of VLFS. Marine 
Struct 2005; 18:149-168.

[6] Ertekin CR, Kim JW, Yoshida K., Mansour AE. (Eds.) Very Large Floating Structures (parts I,II) Mar Struct 2000; Vol 13 No. 4,5 and 2001; Vol 14 No. 1,2.

[7] Kashiwagi, MA. B-spline Galerkin scheme for calculating the hydroelastic response of a very large floating structure waves. J Mar Sci Tech 1998; 3: 3749.

[8] Wang CM, Xiang Y, Utsunomiya T, Watanabe E. Evaluation of modal stress resultants in freely vibrating plates. Int J Solids Struct 2001; 38: 6525-6558.

[9] Chen XJ, Jensen JJ, Cui WC, Fu SX. Hydroelasticity of a floating plate in multidirectional waves. Ocean Eng 2003; 30: 1997-2017.

[10] Eatock Taylor R, Ohkusu M. Green functions for hydroelastic analysis of vibrating freefreebeams and plates. Appl Ocean Res 2000; 22: 295-314.

[11] Kim JW, Ertekin RC. An eigenfunction-expansion method for predicting hydroelastic behaviour of a shallow-draft VLFS. In: Proceedings of the $2^{\text {nd }}$ International Conference on Hydroelastic Marine Technology, Fukuoka, Japan; 1998.

[12] Watanabe E, Utsunomiya T, Tanigaki S. A transient response analysis of a very large floating structure by finite element method. Struct Eng Earthq Eng 1998; 15: $155-163$.

[13] Watanabe E, Utsunomiya T. Transient response analysis of a VLFS at airplane landing. In: Proceedings of the International Workshop on Very Large Floating Structures, Hayama, Japan; 1996. pp. 243-247.

[14] Kashiwagi M. A time-domain mode-expansion method for calculating transient elastic responses of a pontoon-type VLFS. J Mar Sci Technol 2000; 5: 89-100.

[15] Montiel F, Bennets LG, Squire VA. The transient response of floating elastic plates to wavemaker forcing in two dimensions. J Fluid Struct 2012; 28: 416433.

[16] Sturova IV. Time-dependent response of a heterogeneous elastic plate floating on shallow water of variable depth. J Fluid Mech. 2009; 637: 305-325. 
[17] Papathanasiou, TK, Karperaki A, Theotokoglou, EE, Belibassakis, KA. A higher order FEM for time-domain hydroelastic analysis of large floating bodies in an inhomogeneous shallow water environment. Proc Royal Society A 2014; 471: 20140643.

[18] Sun H, Cui WC, Liu YZ, Liao SJ. Hydroelastic response analysis of mat-like VLFS over aplane slope in head seas. China Ocean Eng 2003; 17: 315-326.

[19] Utsunomiya T, Watanabe E, Nishimura N. Fast multipole algorithm for wave diffraction/radiation problems and its application to VLFS in variable water depth and topography. In: Proceedings of the $20^{\text {th }}$ International Conference on Offshore Mechanics and Arctic Engineering; 2001. pp. 1-7.

[20] Belibassakis KA, Athanassoulis GA. A coupled mode model for the hydroelastic analysis of large floating bodies over variable bathymetry regions. J Fluid Mech 2005; 531: 221-249.

[21] Nagata S, Yoshida H, Fujita T, Isshiki H. Reduction of the motion of and elastic floating plate in waves by breakwaters. In: Proceedings of the $2^{\text {nd }}$ International Conference on Hydroelasticity in Marine Technology, Fukuoka, Japan; 1998. pp. $229-238$.

[22] Seto H, Ochi M. A hybrid element approach to hydroelastic behavior of a very large floating structure in regular wave. In: Proceedings of the $2^{\text {nd }}$ International Conference on Hydroelasticity in Marine Technology, Fukuoka, Japan, 1998. pp. 185-194.

[23] Utsunomiya T, Watanabe E, Nakamua N. Analysis of drift force on VLFS by the near-field approach. In: Proceedings of the $11^{\text {th }}$ Offshore \& Polar Engineering Conference, Stavanger, Norway; 2 001. pp. 217-221.

[24] Ohmatsu S. Numerical calculation method of hydroelastic response of a pontoontype VLFS close to a breakwater. In: Proceedings of the 3rd International Workshop VLFS, Honolulu, Hawaii; 1999. pp. 805-811.

[25] Ohta H., Torii T., Hayashi N., Watanabe E., Utsunomiya T., Sekita K., Sunahara S. 1999, Effect of attachment of a horizontal/vertical plate on the wave response of a VLFS. In: Proceedings of the $3^{\text {rd }}$ International Workshop VLFS, Honolulu, Hawaii; 1999. pp. 265-274. 
[26] Watanabe E., Utsunomiya T., Kuramoto M., Ohta H., Torii T., Hayashi N., 2002, Wave response analysis of VLFS with an attached submerged plate. In: Proceedings of the $12^{\text {th }}$ International Conference in Ocean Polar Engineering, Kyushu, Japan; 2002. pp. 319-326.

[27] van Kessel JLF, Pinkster JA. The effect of aircushion division on the motions of large floating structures. In: The proceedings of the $26^{\text {th }}$ International Conference on Offshore Mechanics and Arctic Engineering, San Diego, USA; 2007. pp. 677686.

[28] Khabakhpasheva TI, Korobkin AA. Hydroelastic behaviour of compound floating plate in waves. J Eng Math 2002; 44: 21-40.

[29] Karmakar D, Guedes Soares C, 2012, Scattering of gravity waves by a moored finite floating elastic plate. Appl Ocean Res 2012; 34: 135-149.

[30] Cunbao Z., Jiazhong Z., Wenhu H., Vibration reduction of floating elastic plates in water waves, Marine Structures 2007; 20: 71-99.

[31] Korobkin AA. Khabakhpasheva TI, Wave power absorbers at floating platform In 25th Int. Workshop on Water Waves and Floating Bodies, Harbin, China, 9-12 May 2012 (http://www.iwwwfb.org).

[32] Wu C., Watanabe E. Utsunomiya T., An eigenfunction expansion-matching method for analyzing the wave-induced responses of an elastic floating plate. Appl. Ocean Res. 1995; 17: 301-310.

[33] Stoker J. J. Water Waves: The Mathematical Theory with Applications Interscience 1957.

[34] Karperaki A.E., Belibassakis K.A., Papathanasiou T.K., Markolefas S.I. Higherorder FEM for nonlinear hydroelastic analysis of a floating elastic strip is shallow-water conditions. In: VI International Conference on Computational Methods for Coupled Problems in Science and Engineering, Venice, Italy; 2015. pp. 1110-1022. 